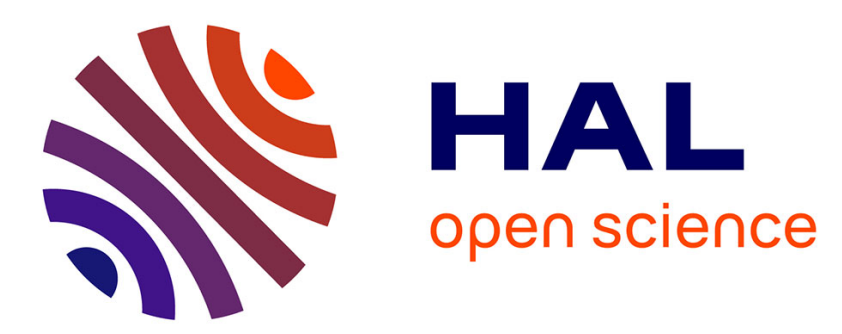

\title{
On the rate of convergence of Schwarz waveform relaxation methods for the time-dependent Schrödinger equation
}

\author{
Xavier Antoine, Emmanuel Lorin
}

\section{To cite this version:}

Xavier Antoine, Emmanuel Lorin. On the rate of convergence of Schwarz waveform relaxation methods for the time-dependent Schrödinger equation. Journal of Computational and Applied Mathematics, 2019, 354, pp.15-30. 10.1016/j.cam.2018.12.006 . hal-01649736

\author{
HAL Id: hal-01649736 \\ https://hal.science/hal-01649736
}

Submitted on 27 Nov 2017

HAL is a multi-disciplinary open access archive for the deposit and dissemination of scientific research documents, whether they are published or not. The documents may come from teaching and research institutions in France or abroad, or from public or private research centers.
L'archive ouverte pluridisciplinaire HAL, est destinée au dépôt et à la diffusion de documents scientifiques de niveau recherche, publiés ou non, émanant des établissements d'enseignement et de recherche français ou étrangers, des laboratoires publics ou privés. 


\title{
On the rate of convergence of Schwarz waveform relaxation methods for the time-dependent Schrödinger equation
}

\author{
X. Antoine $\mathrm{a}^{\mathrm{a}, \mathrm{b}}$, E. Lorin ${ }^{\mathrm{d}, \mathrm{c}}$ \\ ${ }^{a}$ Institut Elie Cartan de Lorraine, Université de Lorraine, F-54506 Vandoeuvre-lès-Nancy Cedex, France \\ ${ }^{b}$ Inria Nancy Grand-Est/IECL - SPHINX team. \\ ${ }^{c}$ Centre de Recherches Mathématiques, Université de Montréal, Montréal, Canada, H3T 1J4 \\ ${ }^{d}$ School of Mathematics and Statistics, Carleton University, Ottawa, Canada, K1S 5B6
}

\begin{abstract}
This paper is dedicated to the analysis of the rate of convergence of the classical and quasioptimal Schwarz waveform relaxation (SWR) method for solving the linear Schrödinger equation with space-dependent potential. The strategy is based on i) the rewriting of the SWR algorithm as a fixed point algorithm in frequency space, and ii) the explicit construction of contraction factors thanks to pseudo-differential calculus. Some numerical experiments illustrating the analysis are also provided.
\end{abstract}

Keywords: Schrödinger equation, domain decomposition method, pseudo-differential calculus.

\section{Introduction}

This paper is devoted to the analysis of the rate of convergence of a class of domain decomposition methods (DDM), the Schwarz Waveform Relaxation algorithms for solving the real-time linear Schrödinger equation (LSE) [3]. The analyzed DDMs provide algorithms for solving all kinds of wave propagation problems, and mainly involve two concepts: relaxation algorithms and well-designed transmission conditions [23].

Let us consider the following initial boundary-value problem: determine the complexvalued wavefunction $u$ solution to the LSE

$$
\begin{aligned}
& \mathrm{i} \partial_{t} u+\triangle u+V(x) u=0, x \in \mathbb{R}, t \geq 0, \\
& |u(x, t)| \rightarrow_{x \rightarrow \pm \infty} 0, t \geq 0, \\
& u(x, 0)=u_{0}(x), x \in \mathbb{R},
\end{aligned}
$$

where $u_{0}$ is the Cauchy data, $V$ is a real-valued space-dependent smooth potential, which is positive (respectively negative) for attractive (respectively repulsive) interactions.

A Schwarz Waveform Relaxation (SWR) domain decomposition algorithm is proposed to Lorin)

Email addresses: xavier.antoine@univ-lorraine.fr (X. Antoine), elorin@math.carleton.ca (E. 
solve the initial value problem (1). In the one-dimensional case, the SWR algorithm decomposes $\mathbb{R}$ into two (or generally more) regions, with or without overlap, and two uncoupled IBVPs are then considered. The SWR iterations allow for a reconstruction of the solution between the adjacent subdomains by exchanging information at the subdomain interfaces thanks to the transmission conditions. This makes these IBVs on each subdomain suitable for parallel computing [15].

In this paper, we analyze the rate of convergence of the Classical and quasi-Optimal Schwarz Waveform Relaxation (CSWR and q-OSWR) DDMs [1, 15, 17, 19, 20, 18, 21, 23, 28], applied to the time-dependent Schrödinger equation in one-dimension. Although these methods have received much attention over the past decades, the first application to the Schrödinger equation can be found in [23], where the authors consider the real-time linear one-dimensional Schrödinger equation. In another recent paper [12], some algorithms are analyzed for the one-dimensional time-dependent Linear Schrödinger Equation (LSE) where are included ionization and recombination processes by an intense electric field, and in [26] a SWR methodology for solving the $N$-body Schrödinger equation is considered. In [14], the authors study the numerical performance with a GPU implementation of Schwarz waveform relaxation methods for the one-dimensional dynamical solution of the LSE with a general potential. It is shown that these algorithms are fast and robust for complex linear problems. In [27], domain decomposition methods have been developed and combined with geometric optics and frozen gaussian approximation for computing the solution to the linear Schrödinger equations under and beyond the semi-classical regime. Finally, [9] is dedicated to the development of high-order transmission conditions for SWR methods applied to the Schrödinger equation, using only local operators.

The goal of the present paper is to contribute to the mathematical understanding of Schwarz waveform relaxation DDMs for solving the time-dependent Schrödinger equation. Although SWR-DDM methods are now extensively used in all kinds of high dimensional problems, the rigorous analysis of the rate of convergence remains incomplete, and is only understood in some simple configurations. This paper is precisely dedicated to this question for the Schrödinger equation in the one-dimensional case with non-constant potentials. The strategy which is proposed can in principle, be applied in higher dimension and in the stationary case, see $[10,11]$. It extensively uses pseudo-differential calculus [2, 24, 29], and was originally developed for deriving and analyzing high-order absorbing boundary conditions for classical and quantum wave equations, as well as diffusion equations [7, 8, 13, 16, 22]. Accurate transmission operators (which will provide fast convergence) can indeed be derived by using asymptotic expansions of pseudo-differential operators (in the sense of classical symbols [24]). However, pseudo-differential calculus is also essential for analyzing the rate of convergence of more elementary DDMs, such as the CSWR and Robin-SWR methods which are respectively based on Dirichlet or Robin transmission operators.

In Subsections 1.1 and 1.2, we introduce preliminary notations, definitions and we recall some existing results about the convergence of SWR methods. The rate of convergence of both the CSWR and the q-OSWR methods with constant and space-dependent potentials $V$, is analyzed in Section 2. The analysis uses pseudo-differential operator theory associated 
to their asymptotic symbolic calculus. In Section 3, we numerically validate the convergence rates theoretically established. We conclude in Section 4.

\subsection{Brief description of the Schwarz Waveform Relaxation algorithm}

In this section, we briefly describe the Classical (resp. quasi-Optimal) Schwarz Waveform Relaxation Algorithm (CSWR) (resp. (q-OSWR)).

We consider a $d$-dimensional partial differential equation $P \phi=f$ in the spatial domain $\Omega \subset \mathbb{R}^{d}$, and time domain $(0, T)$. The initial data is denoted by $\phi_{0}$. We first split $\Omega$ into two subdomains $\Omega_{\varepsilon}^{ \pm}$with smooth boundary, with or without overlap $\left(\Omega_{\varepsilon}^{+} \cap \Omega_{\varepsilon}^{-}=\emptyset\right.$ or $\left.\Omega_{\varepsilon}^{+} \cap \Omega_{\varepsilon}^{-} \neq \emptyset\right)$, with $\epsilon>0$. The CSWR algorithm consists in iteratively solving IBVPs in $\Omega_{\varepsilon}^{ \pm} \times(0, T)$, using Dirichlet transmission conditions at the subdomain interfaces $\Gamma_{\varepsilon}^{ \pm}:=\partial \Omega_{\varepsilon}^{ \pm}$, where the imposed conditions are established using the preceding Schwarz iteration data in the adjacent subdomain. For $k \geq 1$, we therefore set

$$
\begin{cases}P \phi^{ \pm,(k)} & =f, \text { in } \Omega_{\varepsilon}^{ \pm} \times(0, T) \\ \phi^{ \pm,(k)}(\cdot, 0) & =\phi_{0}^{ \pm}, \text {in } \Omega_{\varepsilon}^{ \pm}, \\ \phi^{ \pm,(k)} & =\phi^{ \pm,(k-1)}, \text { on } \Gamma_{\varepsilon}^{ \pm} \times(0, T)\end{cases}
$$

with a given initial guess $\phi^{ \pm,(0)}$. The quasi-Optimal Schwarz Waveform Relaxation algorithm is described in the same manner as the CSWR algorithm, except that transparent or high-order absorbing transmission conditions are imposed thanks to a (pseudo-)differential operator $\mathcal{B}_{ \pm}$, see $[6]$. Consequently, for $k \geq 1$, we define

$$
\left\{\begin{array}{l}
P \phi^{ \pm,(k)}=f, \text { in } \Omega_{\varepsilon}^{ \pm} \times(0, T) \\
\phi^{ \pm,(k)}(\cdot, 0)=\phi_{0}^{ \pm}, \text {in } \Omega_{\varepsilon}^{ \pm} \\
\mathcal{B}_{ \pm}^{(k)} \phi^{ \pm,(k)}=\mathcal{B}_{ \pm}^{(k-1)} \phi^{\mp,(k-1)}, \text { on } \Gamma_{\varepsilon}^{ \pm} \times(0, T)
\end{array}\right.
$$

with a given initial guess $\phi^{ \pm,(0)}$.

\subsection{Well-posedness of the SWR algorithm}

We now define the real time-dependent one-dimensional $(d=1)$ Schrödinger operator $P$ by

$$
P\left(x, t, \partial_{x}, \partial_{t}\right)=\mathrm{i} \partial_{t}+\partial_{x}^{2}+V(x) .
$$

We decompose the spatial domain $\Omega=\mathbb{R}$ into two overlapping subdomains $\Omega_{\varepsilon}^{ \pm}$with $\Omega_{\varepsilon}^{+}=$ $(-\infty, \varepsilon / 2)$ and $\Omega_{\varepsilon}^{-}=(-\varepsilon / 2, \infty)$, with $\varepsilon>0$. The Schwarz waveform relaxation with overlap corresponds to iteratively solving two IBVPs in $\Omega_{\varepsilon}^{ \pm} \times(0, T)$, by using some transmission conditions at the interfaces $x= \pm \varepsilon / 2$. The CSWR algorithm for the iteration index $k=$ $1,2, \ldots$ is thus given by

$$
\begin{cases}P \phi^{ \pm,(k)} & =0, \text { in } \Omega_{\varepsilon}^{ \pm} \times(0, T), \\ \phi^{ \pm,(k)}(\cdot, 0) & =\phi_{0}^{ \pm}, \text {in } \Omega_{\varepsilon}^{ \pm}, \\ \phi^{ \pm,(k)}( \pm \varepsilon / 2, \cdot) & =\phi^{\mp,(k-1)}( \pm \varepsilon / 2, \cdot), \text { on }(0, T),\end{cases}
$$


where $\phi_{0}^{ \pm}$denotes the restriction of $\phi_{0}$ to $\Omega_{\varepsilon}^{ \pm}$. Introducing the error function for arbitrary iteration $k$ and subsequently omitted, i.e. $e_{P}^{C, \pm}:=\phi_{\mid \Omega_{\varepsilon}^{ \pm}}-\phi^{ \pm}$, the CSWR in $L^{2}\left(\Omega_{\varepsilon}^{ \pm}\right)$reads

$$
P e_{P}^{C, \pm}=0 \text { in } \Omega_{\varepsilon}^{ \pm} \times(0, T), \quad e_{P}^{C, \pm}( \pm \varepsilon / 2, t)=h_{\varepsilon}^{ \pm}(t) \text { at }\{ \pm \varepsilon / 2\} \times(0, T),
$$

where $P$ is given by (4) and $h_{\varepsilon}^{ \pm}$are given time-dependent functions. The index $P$ in $e_{P}^{C, \pm}$ specifies the operator to which the error is associated to, and the upper index $C$ stands for the CSWR algorithm (we will later use the upper index $O$ for the q-OSWR algorithm). The following theorem states the convergence of the CSWR algorithm for a bounded potential $V$.

Theorem 1.1. Let $\varepsilon>0, V$ be in $L^{\infty}\left(\Omega_{\varepsilon}^{-} \cup \Omega_{\varepsilon}^{+}\right)$. Then the algorithm (5) defines a sequence of iterates $\left(\phi^{+,(k)}, \phi^{-,(k)}\right)$ in $H^{2,1}\left(\Omega_{\varepsilon / 2}^{+} \times(0, T)\right) \times H^{2,1}\left(\Omega_{\varepsilon}^{-} \times(0, T)\right)$ with $\phi^{+,(k)}(-\varepsilon / 2, \cdot), \partial_{x} \phi^{+,(k)}(-\varepsilon / 2, \cdot), \phi^{-,(k)}(\varepsilon / 2, \cdot)$ and $\partial_{x} \phi^{-,(k)}(\varepsilon / 2, \cdot)$ in $H^{1}(0, T)$.

We recall that $H^{r, s}(\Omega \times(0, T)):=L^{2}\left(0, T ; H^{r}(\Omega)\right) \cap H^{s}\left(0, T ; L^{2}(\Omega)\right)$ is an anisotropic Sobolev space. This result is proven in [23], and is a consequence of the Trace Theorem [25]. In this paper, we are more specifically interested in estimating the convergence rate of the SWR methods. The convergence rate appears as a contraction factor, when the SWR method is rewritten as a fixed point problem. We do not recall here the details of this technical question, but we refer to $[10,17,18]$ and Subsection 2.3. Basically, in the following, we explicitly compute the contraction factor from which we can deduce the rate of convergence of the considered SWR methods.

\section{Convergence rate of Schwarz Waveform Relaxation algorithm}

Considering again the Schrödinger operator $P$ defined in (4), we estimate in this section the theoretical convergence rates of the CSWR and q-OSWR methods. With this aim, we first provide a local factorization of the operator $P$ in term of an incoming and outgoing wave operators.

Proposition 2.1. The Nirenberg-like factorization

$$
P\left(x, t, \partial_{x}, \partial_{t}\right)=\left(\partial_{x}+i \Lambda^{-}\right)\left(\partial_{x}+i \Lambda^{+}\right)+\mathcal{R}
$$

holds, where $\mathcal{R} \in \mathrm{OPS}^{-\infty}=\bigcap_{m} \mathrm{OPS}^{m}$ is a smooth pseudo-differential operator. The operators $\Lambda^{ \pm}$are pseudo-differential operators of order $1 / 2$ in time, and order 0 in space. In addition, their total symbol $\lambda^{ \pm}$can be expanded in $S^{1 / 2}$ as

$$
\lambda^{ \pm} \sim \sum_{j=0}^{\infty} \lambda_{1 / 2-j / 2}
$$

where $\lambda_{1 / 2-j / 2}$ are elementary symbols corresponding to operators of order $1 / 2-j / 2, j \in \mathbb{N}$.

We can approximate $\lambda^{ \pm}$by computing a finite number of inhomogeneous symbols [6]. Denoting by $\tau$ the co-variable associated to $t$, we have: 
Proposition 2.2. Let us fix the principal symbol to

$$
\lambda_{1 / 2}^{ \pm}=\mp \sqrt{-\tau+V(x)} .
$$

Then, the following elementary symbols are given by

$$
\lambda_{0}^{ \pm}=0, \lambda_{-1 / 2}^{ \pm}=0 \text { and } \lambda_{-1}^{ \pm}=\mp \frac{i}{4} \frac{V^{\prime}(x)}{V(x)-\tau} .
$$

The above propositions are proved [6] by using the following recursive formula, for $j \in \mathbb{N}^{*}$

$$
\lambda_{-j / 2}=\frac{1}{2 \lambda_{1 / 2}^{+}}\left(-i \partial_{x} \lambda_{1 / 2-j / 2}^{+}-\sum_{k=1}^{j} \lambda_{-j / 2+k / 2} \lambda_{1 / 2-k / 2}\right) .
$$

The following proposition which is proven in [10], allows for a fine derivation of the transmission conditions in the q-OSWR method.

Proposition 2.3. Let us define the class of symbols $\mathbb{S}_{S}^{1 / 2}$ by

$$
\begin{aligned}
& \mathbb{S}_{S}^{1 / 2}:=\left\{a \sim \sum_{j=0}^{+\infty} a_{1 / 2-j / 2} \in S_{S}^{1 / 2} \text { such that: } a_{1 / 2-j / 2}(x, \tau):=\right. \\
&\left.\frac{1}{\left(\lambda_{1 / 2}^{+}\right)^{j-1}} \sum_{\ell=0}^{L_{j}} \frac{\mathcal{F}_{\ell}^{V, 1 / 2-j / 2}}{\left(\lambda_{1 / 2}^{+}\right)^{\ell}}, \text { with } \mathcal{F}_{\ell}^{V, 1 / 2-j / 2} \in \mathcal{C}^{\infty}(\mathbb{R} ; \mathbb{R}), L_{j} \in \mathbb{N}\right\},
\end{aligned}
$$

and the associated class of pseudo-differential operators $O P \mathbb{S}_{S}^{1 / 2}$. In $(12), \mathcal{F}_{\ell}^{V, 1 / 2-j / 2}$ are smooth functions depending on $x$ and $V$. Then, $\Lambda^{ \pm}$are in $O P \mathbb{S}_{S}^{1 / 2}$ and, for each $j \in \mathbb{N}$, there exist some regular functions $\left\{\mathcal{F}_{\ell}^{V, 1 / 2-j / 2}\right\}_{\ell=0}^{L_{j}}$ such that

$$
\lambda_{1 / 2-j / 2}^{+}=-\lambda_{1 / 2-j / 2}^{-}=\frac{1}{\left(\lambda_{1 / 2}^{+}\right)^{j-1}} \sum_{\ell=0}^{L_{j}} \frac{\mathcal{F}_{\ell}^{V, 1 / 2-j / 2}}{\left(\lambda_{1 / 2}^{+}\right)^{\ell}} .
$$

\subsection{Asymptotic estimates of the contraction factor of the CSWR algorithm}

In this subsection, we analyze the convergence rate of the CSWR algorithm described in (5). As proposed in [17], we are required to determine the contraction factor $C_{P, \varepsilon}^{C}$ of $\mathcal{G}_{P}^{C 2}$ (setting $\mathcal{G}_{P}^{C 2}:=\mathcal{G}_{P}^{C} \circ \mathcal{G}_{P}^{C}$ ), where the mapping $\mathcal{G}_{P}^{C}$ is defined from (6) by

$$
\mathcal{G}_{P}^{C}:\left\langle h_{\varepsilon}^{+}, h_{\varepsilon}^{-}\right\rangle \mapsto\left\langle e_{P}^{C,-}(\varepsilon / 2, \cdot), e_{P}^{C,+}(-\varepsilon / 2, \cdot)\right\rangle .
$$

The time-dependent functions $h_{\varepsilon}^{ \pm}$are assumed to be given. In order to simplify the notation, we denote by $h_{\varepsilon}^{ \pm}$the extension of $h_{\varepsilon}^{ \pm}$to all $\mathbb{R}$ which vanishes on $\Omega_{\varepsilon}^{\mp}$. We solve (6) directly to prove that $\mathcal{G}_{P}^{C 2}$ is a contraction in the $(x, \tau)$-coordinates for $V$ constant. For a nonconstant $V$, we estimate the rate of convergence through approximations. Let us then start 
by assuming that $V$ is a constant (this includes $V=0$ ). According to [17], for a fixed time $T, \mathcal{G}_{P}^{C}$ is defined on $H_{0}^{3 / 4}(0, T)=\left\{\phi \in H^{3 / 4}(0, T): \phi(0)=0\right\}$. Let us characterize the part of the error $e_{P}^{C,+}$ (resp. $e_{P}^{C,-}$ ) defined in (6), corresponding to a traveling wave in the overlapping region $\Omega_{\varepsilon}^{+}$(resp. $\Omega_{\varepsilon}^{-}$) and transmitted to the left (resp. right) subdomain $\mathbb{R} / \bar{\Omega}_{\varepsilon}^{+}$ (resp. $\mathbb{R} / \bar{\Omega}_{\varepsilon}^{-}$). Therefore, we introduce the equation

$$
\left\{\begin{array}{l}
\left(\partial_{x}+i \Lambda^{\mp}\right) e_{\Lambda}^{C, \pm}=0, \text { in } \Omega_{\varepsilon}^{ \pm} \\
e_{\Lambda}^{C, \pm}( \pm \varepsilon / 2, t)=h_{\varepsilon}^{ \pm}(t) \text { at }\{ \pm \varepsilon / 2\} \times \mathbb{R} .
\end{array}\right.
$$

For $V=0$, the solution to (15) can be explicitly computed using the Fourier transform $\mathcal{F}_{t}$ along the $t$-direction, that is at the symbol level with respect to $\tau$ (associated to $t$ ). This exact solution to $(15)$ is given in the $(x, \tau)$-space by

$$
\hat{e}_{\Lambda}^{C, \pm}(x, \tau)=\widehat{h}_{\varepsilon}^{ \pm}(\tau) \exp \left(-\mathrm{i} \int_{ \pm \varepsilon / 2}^{x} \lambda^{\mp}(\tau) d y\right) .
$$

The application of Proposition (2.2) for $V$ constant leads to: $\lambda^{ \pm}(\tau)=\lambda_{1 / 2}^{ \pm}(\tau)=\mp \sqrt{-\tau+V}$. Then, if we define

$$
\mathcal{G}_{\Lambda}^{C}:\left\langle h_{\varepsilon}^{+}, h_{\varepsilon}^{-}\right\rangle \mapsto\left\langle e_{\Lambda}^{C,-}(\varepsilon / 2, \cdot), e_{\Lambda}^{C,+}(-\varepsilon / 2, \cdot)\right\rangle,
$$

we have

$$
\begin{aligned}
& \mathcal{F}_{t}\left(\mathcal{G}_{\Lambda}^{C 2}\left\langle h_{\varepsilon}^{+}, h_{\varepsilon}^{-}\right\rangle\right) \\
& =\left\langle\exp \left(\mathrm{i} \int_{-\varepsilon / 2}^{\varepsilon / 2}\left(\lambda^{-}(\tau)-\lambda^{+}(\tau)\right) d y\right) \hat{h}_{\varepsilon}^{+}, \exp \left(\mathrm{i} \int_{-\varepsilon / 2}^{\varepsilon / 2}\left(\lambda^{-}(\tau)-\lambda^{+}(\tau)\right) d y\right) \hat{h}_{\varepsilon}^{-}\right\rangle \\
& =\exp \left(-2 \mathrm{i} \varepsilon \lambda^{+}(\tau)\right)\left\langle\hat{h}_{\varepsilon}^{+}, \hat{h}_{\varepsilon}^{-}\right\rangle .
\end{aligned}
$$

Following [17,6], we deduce the contraction factors $C_{\Lambda, \varepsilon}^{C}$ of $\mathcal{G}_{\Lambda}^{C 2}$ in the elliptic and hyperbolic zones. The convergence of the CSWR method is ensured by the frequencies in the elliptic zones, as the frequencies in the hyperbolic zone do not affect the convergence process. In the following, we mainly restrict the analysis of the contraction factor to the elliptic zone. We denote by $C_{P, \varepsilon}^{C}$ the contraction factor of $\mathcal{G}_{P}^{C 2}$ in the elliptic zone. We get:

$$
C_{P, \varepsilon}^{C}=C_{\Lambda, \varepsilon}^{C}=\sup _{\tau \in \mathcal{E}_{\tau}} L_{\Lambda, \varepsilon}^{C}(\tau),
$$

where

$$
L_{\Lambda, \varepsilon}^{C}(\tau)=\left|\exp \left(-2 \mathbf{i} \varepsilon \lambda^{+}(\tau)\right)\right|=\exp (-2 \varepsilon \sqrt{\tau-V}) .
$$

In the above expression the elliptic zone is denoted by $\mathcal{E}_{\tau}=\{\tau \in \mathbb{R}: \tau>V\}$, and $\mathcal{H}_{\tau}$ is the hyperbolic zone $\{\tau \in \mathbb{R}: \tau<V\}$. It is also noteworthy that it is well-known that the 
CSWR does not converge without overlap, i.e. when $\varepsilon=0$.

We now consider the space-dependent potential $V(x)$, where the hyperbolic (resp. elliptic) zone is now defined by $\{\tau \in \mathbb{R}: \tau<V(x)\}$ (resp. $\{\tau \in \mathbb{R}: \tau>V(x)\}$ ). We need to characterize the error that travels from one domain to the other. For a general potential $V(x)$, the analysis cannot be exact due to scattering effects. However, we still consider the system

$$
\left\{\begin{array}{l}
\left(\partial_{x}+i \Lambda^{\mp}\right) e_{\Lambda}^{C, \pm}=0, \text { in } \Omega_{\varepsilon}^{ \pm}, \\
e_{\Lambda}^{C, \pm}( \pm \varepsilon / 2, t)=h_{\varepsilon}^{ \pm}(t) \text { at }\{ \pm \varepsilon / 2\} \times \mathbb{R},
\end{array}\right.
$$

where $e_{\Lambda}^{C,+}$ (resp. $e_{\Lambda}^{C,-}$ ) is to be understood as the part of $e_{P}^{C,+}$ (resp. $e_{P}^{C,-}$ ) which travels to the right (resp. left). As a consequence, the computation of $e_{\Lambda}^{C, \pm}$ provides an approximation of $e_{P}^{C, \pm}$ the solution to $P e_{P}^{C, \pm}=0$. We can obtain an approximation of the contraction factor $C_{P, \varepsilon}^{C}$, which is the contraction factor of $\mathcal{G}_{P}^{C 2}$ by $C_{\Lambda, \varepsilon}^{C}$ for $\mathcal{G}_{\Lambda}^{C 2}$

$$
C_{P, \varepsilon}^{C} \approx C_{\Lambda, \varepsilon}^{C}
$$

This approximation will be discussed at the end of this subsection. For solving (19), let us consider the symbolic equation

$$
\left\{\begin{array}{l}
\left(\partial_{x}+i \lambda^{\mp}(x, \tau)\right) \widehat{e}_{\Lambda}^{C, \pm}(x, \tau)=0, \text { in } \Omega_{\varepsilon}^{ \pm}, \\
\widehat{e}_{\Lambda}^{C, \pm}( \pm \varepsilon / 2, \tau)=\widehat{h}_{\varepsilon}^{ \pm}(\tau) \text { at }\{ \pm \varepsilon / 2\} \times \mathbb{R} .
\end{array}\right.
$$

A direct computation gives

$$
\hat{e}_{\Lambda}^{C, \pm}(x, \tau)=\widehat{h}_{\varepsilon}^{ \pm}(\tau) \exp \left(-i \int_{ \pm \varepsilon / 2}^{x} \lambda^{\mp}(y, \tau) d y\right) .
$$

To determine the contraction factor $C_{\Lambda, \varepsilon}^{C}$ to the associated mapping $\mathcal{G}_{\Lambda}^{C 2}$, the explicit knowledge of the total symbols $\lambda^{ \pm}$is required. However, for a general potential $V(x)$, this is generally impossible. We circumvent this issue by considering an asymptotic expansion $\left\{\lambda_{1 / 2-j / 2}^{ \pm}\right\}_{j=0}^{+\infty}$ of the symbols $\lambda^{ \pm}$. To get this estimate, we expand $\lambda^{ \pm}$asymptotically, as the sum of elementary inhomogeneous symbols

$$
\lambda^{ \pm} \sim \sum_{j=0}^{ \pm \infty} \lambda_{1 / 2-j / 2}^{ \pm}
$$

and then we truncate this series up to the $(p+1)^{\text {st }}$ term

$$
\lambda^{ \pm} \approx \lambda^{ \pm, p}=\sum_{j=0}^{p} \lambda_{1 / 2-j / 2}^{ \pm}
$$

as proposed in [4]. The sign $\approx$ has to be understood as a truncation of the infinite series by a finite sum, up to a symbol of homogeneity degree $-p / 2$ in $\tau$. In other words, for $|\tau|$ large 
enough, we truncate up to a $O\left(\tau^{-p / 2}\right)$. The approximate convergence rate in the elliptic zone is then

$$
C_{P, \varepsilon}^{C} \approx C_{\Lambda, \varepsilon}^{C} \approx C_{\varepsilon}^{C, p}:=\sup _{\tau \in \mathcal{E}_{\tau}} L_{\varepsilon}^{C, p}(\tau)
$$

with

$$
L_{\varepsilon}^{C, p}(\tau)=\left|\exp \left(i \int_{-\varepsilon / 2}^{\varepsilon / 2}\left(\lambda^{-, p}(y, \tau)-\lambda^{+, p}(y, \tau)\right) d y\right)\right| .
$$

Choosing the principal symbol as $\lambda_{1 / 2}^{ \pm}=\mp \sqrt{-\tau+V(x)}$, then one gets

$$
\lambda^{-, p}=-\lambda^{+, p},
$$

implying that (23) becomes

$$
L_{\varepsilon}^{C, p}(\tau)=\left|\exp \left(-2 \mathrm{i} \int_{-\varepsilon / 2}^{\varepsilon / 2} \lambda^{+, p}(y, \tau) d y\right)\right| .
$$

Let us remark that (24) does not hold for $\lambda_{1 / 2}^{ \pm}=\mp \sqrt{-\tau}$, if $V(x) \neq 0$ [6]. A third step is required to approximate the symbols $\lambda_{1 / 2-j / 2}^{ \pm}, j=0, \ldots, p$, where $1 /|\tau|$ is small (highfrequency regime). For each symbol $\lambda_{1 / 2-j / 2}^{ \pm}$, we consider a Taylor expansion up to the order $p / 2$ in $1 /|\tau|:$

$$
\lambda^{ \pm, p} \approx \widetilde{\lambda}^{ \pm, p}=\sum_{j=0}^{p}\left(\lambda_{1 / 2-j / 2}^{ \pm}\right)_{(1-p) / 2}
$$

where

$$
\left(\lambda_{1 / 2-j / 2}^{ \pm}\right)_{(1-p) / 2}=\lambda_{1 / 2-j / 2}^{ \pm}+O\left(|\tau|^{-p / 2}\right)
$$

Notice that Padé's approximants could also be used to approximate $\lambda_{1 / 2-j / 2}^{ \pm}$without requiring $1 /|\tau|$ small, see $[6]$. We then define

$$
\widetilde{L}_{\varepsilon}^{C, p}(\tau)=\left|\exp \left(-2 \mathrm{i} \int_{-\varepsilon / 2}^{\varepsilon / 2} \tilde{\lambda}^{+, p}(y, \tau) d y\right)\right|,
$$

and the associated high-frequency asymptotic convergence rate in the elliptic zone $\widetilde{C}_{\varepsilon}^{C, p}$, where

$$
C_{P, \varepsilon}^{C} \approx C_{\Lambda, \varepsilon}^{C} \approx \widetilde{C}_{\varepsilon}^{C, p}:=\sup _{\tau \in \mathcal{E}_{\tau}} \widetilde{L}_{\varepsilon}^{C, p}(\tau)
$$

Let us now set

$$
\begin{aligned}
& L_{\varepsilon, 1 / 2-j / 2}(\tau)=\left|\exp \left(-2 i \int_{-\varepsilon / 2}^{\varepsilon / 2} \lambda_{1 / 2-j / 2}^{+}(y, \tau) d y\right)\right| \\
& \widetilde{L}_{\varepsilon, 1 / 2-j / 2}^{p}(\tau)=\left|\exp \left(-2 i \int_{-\varepsilon / 2}^{\varepsilon / 2}\left(\lambda_{1 / 2-j / 2}^{+}\right)_{(1-p) / 2}(y, \tau) d y\right)\right| .
\end{aligned}
$$


Therefore, we have

$$
L_{\varepsilon}^{C, p}=\prod_{j=0}^{p} L_{\varepsilon, 1 / 2-j / 2} \text { and } \widetilde{L}_{\varepsilon}^{C, p}=\prod_{j=0}^{p} \widetilde{L}_{\varepsilon, 1 / 2-j / 2}^{p}
$$

These preliminary computations lead to the following theorem.

Theorem 2.1. Let $V(x)$ be a smooth potential, and let us assume that the symbols $\left\{\lambda_{1 / 2-j / 2}^{ \pm}\right\}_{j \geq 0}$ are defined as in Proposition 2.2. An asymptotic estimate in the elliptic zone $\mathcal{E}_{\tau}$ of the contraction factor of the mapping $\mathcal{G}_{P}^{C 2}$ defined in (14) for the CSWR algorithm (5), is given by

$$
C_{P, \varepsilon}^{C} \approx C_{\varepsilon}^{C, 3}=\sup _{\tau \in \mathcal{E}_{\tau}} L_{\varepsilon}^{C, 3}(\tau)
$$

with

$$
L_{\varepsilon}^{C}(\tau) \approx L_{\varepsilon}^{C, 3}(\tau):=\left|\left(\frac{-\tau+V(-\varepsilon / 2)}{-\tau+V(+\varepsilon / 2)}\right)^{1 / 2}\right|\left|\exp \left(-2 \mathrm{i} \int_{-\varepsilon / 2}^{\varepsilon / 2} \sqrt{-\tau+V(y)} d y\right)\right|
$$

while in the hyperbolic zone $\mathcal{H}_{\tau}$ the contraction factor is given by the first term in (31). In addition, one also gets the following approximation of the contraction factor in the elliptic zone, when the symbols are Taylorized for $|\tau|$ large

$$
C_{P, \varepsilon}^{C} \approx \widetilde{C}_{\varepsilon}^{C, 3}=\sup _{\tau \in \mathcal{E}_{\tau}} \widetilde{L}_{\varepsilon}^{C, 3}(\tau)
$$

with

$$
L_{\varepsilon}^{C}(\tau) \approx \widetilde{L}_{\varepsilon}^{C, 3}(\tau):=\exp \left(-2 \varepsilon \sqrt{-\tau}+\frac{1}{2 \sqrt{-\tau}} \int_{-\varepsilon / 2}^{\varepsilon / 2} V(y) d y\right)
$$

Proof. From (21), we have

$$
\hat{e}_{\Lambda}^{C, \pm}(x, \tau)=\widehat{h}_{\varepsilon}^{ \pm}(\tau) \exp \left(-\mathrm{i} \int_{ \pm \varepsilon / 2}^{x} \lambda^{\mp}(y, \tau) d y\right) .
$$

This implies that

$$
\mathcal{F}_{t}\left(\mathcal{G}_{P}^{C 2}\left\langle h_{\varepsilon}^{+}, h_{\varepsilon}^{-}\right\rangle\right) \approx \exp \left(i \int_{-\varepsilon / 2}^{\varepsilon / 2}\left(\lambda^{-}(y, \tau)-\lambda^{+}(y, \tau)\right) d y\right)\left\langle\hat{h}_{\varepsilon}^{+}, \hat{h}_{\varepsilon}^{-}\right\rangle .
$$

By using Proposition 2.2, one gets

$$
\lambda_{1 / 2}^{ \pm}(x, \tau)=\mp \sqrt{-\tau+V(x)}, \quad \lambda_{-1}^{ \pm}(x, \tau)=\mp \frac{1}{4-\tau+V(x)},
$$


$\lambda_{0}^{ \pm}=0$ and $\lambda_{-1 / 2}^{ \pm}(x, \tau)=0$. A direct computation leads to

$$
\begin{aligned}
& L_{\varepsilon, 1 / 2}(\tau)=\left|\exp \left(-2 \mathrm{i} \int_{-\varepsilon / 2}^{\varepsilon / 2} \sqrt{-\tau+V(y)} d y\right)\right|, \\
& L_{\varepsilon,-1}(\tau)=\left|\exp \left(\frac{\mathrm{i}}{2} \int_{-\varepsilon / 2}^{\varepsilon / 2} \frac{V^{\prime}(y)}{-\tau+V(y)} d y\right)\right|
\end{aligned}
$$

with $L_{\varepsilon, 0}=L_{\varepsilon,-1 / 2}=1$, and

$$
L_{\varepsilon,-1}(\tau)=\left|\left(\frac{-\tau+V(-\varepsilon / 2)}{-\tau+V(+\varepsilon / 2)}\right)^{1 / 2}\right|
$$

As a consequence, one gets

$$
L_{\varepsilon}^{C, 3}(\tau)=\left|\left(\frac{-\tau+V(-\varepsilon / 2)}{-\tau+V(+\varepsilon / 2)}\right)^{1 / 2} \| \exp \left(-2 \mathbf{i} \int_{-\varepsilon / 2}^{\varepsilon / 2} \sqrt{-\tau+V(y)} d y\right)\right| .
$$

We obtain

$$
\left(\lambda_{1 / 2}^{+}\right)_{-1}=\sqrt{-\tau}\left(1-\frac{V(x)}{2 \tau}\right)
$$

and then $\widetilde{L}_{\varepsilon, 0}^{3}=\widetilde{L}_{\varepsilon,-1 / 2}^{3}=\widetilde{L}_{\varepsilon,-1}^{3}=1$,

$$
\widetilde{L}_{\varepsilon, 1 / 2}^{3}=\left|\exp \left(-2 \varepsilon \sqrt{-\tau}-\frac{1}{2 \sqrt{-\tau}} \int_{-\varepsilon / 2}^{\varepsilon / 2} V(y) d y\right)\right|
$$

The proof follows from $\widetilde{L}_{\varepsilon}^{C, 3}=\widetilde{L}_{\varepsilon, 1 / 2}^{3}$.

By neglecting the scattering effects in (6), we have then estimated in the above theorem the approximate rate of convergence for the CSWR method with non-constant potentials. In the following proposition, we estimate $\left|C_{P, \varepsilon}^{C}-C_{\Lambda, \varepsilon}^{C}\right|$ assuming that $V$ and $V^{\prime}$ are bounded.

Proposition 2.4. With the same assumptions as in Theorem 2.1, and assuming that $V$ and $V^{\prime}$ are in $L^{\infty}\left(\Omega_{\varepsilon}^{ \pm}\right)$, there exists $C(\varepsilon, V)>0$ such that for $\varepsilon$ small enough:

$$
C_{P, \varepsilon}^{C} \leq C_{\Lambda, \varepsilon}^{C}+C(\varepsilon, V) \varepsilon^{2}
$$

This result ensures that if $V$ and $V^{\prime}$, and/or $\varepsilon$ are small enough, the exact contract factor $C_{P, \varepsilon}^{C}$, is close to the approximate one $C_{\Lambda, \varepsilon}^{C}$ computed in Theorem 2.1. This expected result is confirmed numerically in Section 3.

Proof. Recall that $\widehat{e}_{P}^{C, \pm}$ (resp. $\widehat{e}_{\Lambda}^{C, \pm}$ ) denotes the solution in $L^{2}\left(\Omega_{\varepsilon}^{ \pm}\right)$to (6) (resp. to (15)). For the sake of notation simplicity, we hereafter omit in the proof the upper index $C$ in $\widehat{e}_{P}^{C, \pm}$ 
and $\widehat{e}_{\Lambda}^{C, \pm}$. We now set $f^{ \pm}:=e_{P}^{ \pm}-e_{\Lambda}^{ \pm}$. Some basic computations show that $\widehat{f}^{ \pm}$is solution in $L^{2}\left(\Omega_{\varepsilon}^{ \pm}\right)$to

$$
\left\{\begin{array}{l}
\left(\partial_{x}^{2}-(\mathrm{i} \tau+V(x))\right) \widehat{f}^{ \pm}(x, \tau)=\mathrm{i} \partial_{x} \lambda^{ \pm} \widehat{e}_{\Lambda}^{ \pm}, \text {in } \Omega_{\varepsilon}^{ \pm} \\
\hat{f}^{ \pm}( \pm \varepsilon / 2, \tau)=0, \text { at }\{ \pm \varepsilon / 2\} \times \mathbb{R}
\end{array}\right.
$$

As $V$ and $V^{\prime}$ are assumed to be in $L^{\infty}\left(\Omega_{\varepsilon}^{ \pm}\right)$, then $\widehat{e}_{\Lambda}^{ \pm}$(resp. $\widehat{f}^{ \pm}$) for any fixed $\tau$, belongs to $C^{1}\left(\Omega_{\varepsilon}^{ \pm}\right)$(resp. to $C^{2}\left(\Omega_{\varepsilon}^{ \pm}\right)$). Multiplying the first equation of $(39)$ by $\overline{\widehat{f}^{ \pm}}(x, \tau)$, we get

$$
\begin{aligned}
\int_{\Omega_{\varepsilon}^{ \pm}} \overline{\widehat{f}^{ \pm}}(x, \tau) \partial_{x}^{2} \widehat{f}^{ \pm}(x, \tau) d x= & \int_{\Omega_{\varepsilon}^{ \pm}}(\mathbf{i} \tau+V(x))\left|\widehat{f}^{ \pm}(x, \tau)\right|^{2} \\
& +\mathrm{i} \partial_{x} \lambda^{ \pm}(x, \tau) \widehat{e}_{\Lambda}^{ \pm}(x, \tau) \widehat{f}^{ \pm}(x, \tau) d x
\end{aligned}
$$

Taking the imaginary part in (40), we have

$$
|\tau| \int_{\Omega_{\varepsilon}^{ \pm}}\left|\widehat{f}^{ \pm}(x, \tau)\right|^{2} d x=\left|\operatorname{Im}\left\{i \int_{\Omega_{\varepsilon}^{ \pm}} \partial_{x} \lambda^{ \pm}(x, \tau) \widehat{e}_{\Lambda}^{ \pm}(x, \tau) \overline{\widehat{f}^{ \pm}}(x, \tau) d x\right\}\right| .
$$

Then for any $\eta>0$, we obtain

$$
|\tau|\left\|\widehat{f}^{ \pm}(\cdot, \tau)\right\|_{L^{2}\left(\Omega_{\varepsilon}^{ \pm}\right)}^{2} \leq\left\|\partial_{x} \lambda^{ \pm}(\cdot, \tau)\right\|_{L^{\infty}\left(\Omega_{\varepsilon}^{ \pm}\right)}\left(\frac{\eta}{2}\left\|\widehat{f}^{ \pm}(\cdot, \tau)\right\|_{L^{2}\left(\Omega_{\varepsilon}^{ \pm}\right)}^{2}+\frac{1}{2 \eta}\left\|e_{\Lambda}^{ \pm}(\cdot, \tau)\right\|_{L^{2}\left(\Omega_{\varepsilon}^{ \pm}\right)}^{2}\right)
$$

By taking $\eta$ small enough, we deduce that $\widehat{f}^{ \pm}(\cdot, \tau) \in L^{2}\left(\Omega_{\varepsilon}^{ \pm}\right)$. Then taking the real part in (40) for any $\eta>0$, we get

$$
\begin{aligned}
\left\|\partial_{x} \widehat{f}^{ \pm}(\cdot, \tau)\right\|_{L^{2}\left(\Omega_{\varepsilon}^{ \pm}\right)}^{2} \leq & \left\|\partial_{x} \lambda^{ \pm}(\cdot, \tau)\right\|_{L^{\infty}\left(\Omega_{\varepsilon}^{ \pm}\right)}\left(\frac{\eta}{2}\left\|\widehat{f}^{ \pm}(\cdot, \tau)\right\|_{L^{2}\left(\Omega_{\varepsilon}^{ \pm}\right)}^{2}+\frac{1}{2 \eta}\left\|e_{\Lambda}^{ \pm}(\cdot, \tau)\right\|_{L^{2}\left(\Omega_{\varepsilon}^{ \pm}\right)}^{2}\right) \\
& +\|V\|_{L^{\infty}\left(\Omega_{\varepsilon}^{ \pm}\right)}\left\|\widehat{f}^{ \pm}(\cdot, \tau)\right\|_{L^{2}\left(\Omega_{\varepsilon}^{ \pm}\right)}^{2}
\end{aligned}
$$

Similarly, multiplying by $\partial_{x}^{2} \widehat{\widehat{f}}^{ \pm}(x, \tau)$, we have

$$
\begin{aligned}
\int_{\Omega_{\varepsilon}^{ \pm}}\left|\partial_{x}^{2} \widehat{f}^{ \pm}(x, \tau)\right|^{2} d x= & -\int_{\Omega_{\varepsilon}^{ \pm}}\left((\mathrm{i} \tau+V(x))\left|\partial_{x} \widehat{f}^{ \pm}(x, \tau)\right|^{2}+V^{\prime}(x) \widehat{f}^{ \pm}(x, \tau) \partial_{x} \overline{\widehat{f}^{ \pm}}(x, \tau)\right) d x \\
& +\mathrm{i} \int_{\Omega_{\varepsilon}^{ \pm}} \partial_{x} \lambda^{ \pm}(x, \tau) \widehat{e}_{\Lambda}^{ \pm}(x, \tau) \overline{\partial_{x}^{2} \widehat{f}^{ \pm}}(x, \tau) d x
\end{aligned}
$$

and then, for any $\eta>0$,

$$
\begin{aligned}
\left\|\partial_{x}^{2} \widehat{f}^{ \pm}(\cdot, \tau)\right\|^{2} \leq & \left(\|V\|_{L^{\infty}\left(\Omega_{\varepsilon}^{ \pm}\right)}+\left\|V^{\prime}\right\|_{L^{\infty}\left(\Omega_{\varepsilon}^{ \pm}\right)}\right)\left\|\partial_{x} \widehat{f}^{ \pm}(\cdot, \tau)\right\|^{2}+\left\|V^{\prime}\right\|_{L^{\infty}\left(\Omega_{\varepsilon}^{ \pm}\right)}\left\|\widehat{f}^{ \pm}(\cdot, \tau)\right\|^{2} \\
& +\left\|\partial_{x} \lambda^{ \pm}(\cdot, \tau)\right\|_{L^{\infty}\left(\Omega_{\varepsilon}^{ \pm}\right)}\left(\frac{\eta}{2}\left\|\partial_{x}^{2} \widehat{f}^{ \pm}(\cdot, \tau)\right\|_{L^{2}\left(\Omega_{\varepsilon}^{ \pm}\right)}^{2}\left\|+\frac{1}{2 \eta}\right\| e_{\Lambda}^{ \pm}(\cdot, \tau) \|_{L^{2}\left(\Omega_{\varepsilon}^{ \pm}\right)}^{2}\right) .
\end{aligned}
$$

By taking $\eta$ small enough, we deduce that for $\tau$ fixed, $f^{ \pm}(\cdot, \tau) \in H^{2}\left(\Omega_{\varepsilon}^{ \pm}\right)$. Next, we have

$$
\mathcal{F}_{t}\left(\mathcal{G}_{\Lambda}^{C} \circ \mathcal{G}_{\Lambda}^{C}\left\langle h_{\varepsilon}^{+}, h_{\varepsilon}^{-}\right\rangle\right)=\exp \left(i \int_{-\varepsilon / 2}^{\varepsilon / 2}\left(\lambda^{-}(y, \tau)-\lambda^{+}(y, \tau)\right) d y\right)\left\langle\widehat{h}_{\varepsilon}^{+}, \widehat{h}_{\varepsilon}^{-}\right\rangle
$$


as

$$
\widehat{e}_{\Lambda}^{ \pm}(x, \tau)=\widehat{h}_{\varepsilon}^{ \pm}(\tau) \exp \left(-i \int_{ \pm \varepsilon / 2}^{x} \lambda^{\mp}(y, \tau) d y\right)
$$

Now we need to evaluate $\mathcal{F}_{t}\left(\mathcal{G}_{P}^{C} \circ \mathcal{G}_{P}^{C}\left\langle h_{\varepsilon}^{+}, h_{\varepsilon}^{-}\right\rangle\right)$. As $\widehat{f}^{ \pm}$is regular in $x$, by Taylor's expansion and for some $\xi_{x, \varepsilon}^{ \pm}$between $x$ and $\pm \varepsilon / 2$, we have

$$
\begin{aligned}
\widehat{f}^{ \pm}(x, \tau) & =\widehat{f}^{ \pm}( \pm \varepsilon / 2, \tau)+\partial_{x} \widehat{f}^{ \pm}\left(\xi_{x, \varepsilon}^{ \pm}, \tau\right)(x \mp \varepsilon / 2) \\
& =h_{\varepsilon}^{ \pm}(\tau) \partial_{x} \widehat{g}^{ \pm}\left(\xi_{x, \varepsilon}^{ \pm}, \tau\right)(x \mp \varepsilon / 2)
\end{aligned}
$$

as $\widehat{f}^{ \pm}( \pm \varepsilon / 2, \tau)=0$ and as by definition of $\widehat{f}^{ \pm}, \partial_{x} \widehat{f}^{ \pm}$is of the form $h_{\varepsilon}^{ \pm}(\tau) \partial_{x} \widehat{g}^{ \pm}$for some regular function $\widehat{g}^{ \pm}$, i.e.

$$
\widehat{e}_{P}^{ \pm}(x, \tau)=\widehat{e}_{\Lambda}^{ \pm}(x, \tau)+\widehat{h}_{\varepsilon}^{ \pm}(\tau) \partial_{x} \widehat{g}^{ \pm}\left(\xi_{x, \varepsilon}^{ \pm}, \tau\right)(x \pm \varepsilon / 2) .
$$

Now, we can write that

$$
\begin{aligned}
\mathcal{F}_{t}\left(\mathcal{G}_{P}^{C} \circ \mathcal{G}_{P}^{C}\left\langle h_{\varepsilon}^{+}, h_{\varepsilon}^{-}\right\rangle\right)= & \left\langle\widehat{e}_{P}(\varepsilon / 2, \tau), \widehat{e}_{P}^{+}(-\varepsilon / 2, \tau)\right\rangle \\
= & \left\langle\widehat{h}_{\varepsilon}^{-}(\tau) \exp \left(-i \int_{-\varepsilon / 2}^{\varepsilon / 2} \lambda^{+}(y, \tau) d y\right)+\varepsilon \partial_{x} \widehat{f}^{-}\left(\xi_{\varepsilon / 2, \varepsilon}^{-}, \tau\right),\right. \\
& \left.\widehat{h}_{\varepsilon}^{+}(\tau) \exp \left(-i \int_{\varepsilon / 2}^{-\varepsilon / 2} \lambda^{-}(y, \tau) d y\right)-\varepsilon \partial_{x} \widehat{f}^{+}\left(\xi_{-\varepsilon / 2, \varepsilon}^{+}, \tau\right)\right\rangle \\
= & \left\langle\widehat{h}_{\varepsilon}^{+,(2)}(\tau), \widehat{h}_{\varepsilon}^{-,(2)}(\tau)\right\rangle .
\end{aligned}
$$

We iterate once more

$$
\widehat{e}_{P}^{ \pm,(2)}(\mp \varepsilon / 2, \tau)=\widehat{h}_{\varepsilon}^{ \pm,(2)}(\tau) \exp \left(i \int_{\mp \varepsilon / 2}^{ \pm \varepsilon / 2} \lambda^{\mp}(y, \tau) d y\right) \mp \varepsilon \partial_{x} \widehat{f}^{ \pm}\left(\xi_{\mp \varepsilon / 2, \varepsilon}^{ \pm}, \tau\right) .
$$

Some additional computations lead to

$$
\begin{aligned}
\widehat{e}_{P}^{ \pm,(2)}(\mp \varepsilon / 2, \tau)= & \widehat{h}_{\varepsilon}^{\mp}(\tau) \exp \left(-2 i \int_{-\varepsilon / 2}^{\varepsilon / 2} \lambda^{+}(y, \tau) d y\right) \\
& \pm \varepsilon\left(\exp \left(-i \int_{-\varepsilon / 2}^{\varepsilon / 2} \lambda^{+}(y, \tau) d y\right)\right. \\
& \left.\times \partial_{x} \widehat{f}^{\mp}\left(\xi_{ \pm \varepsilon / 2, \varepsilon}^{\mp}, \tau\right)-\partial_{x} \widehat{f}^{ \pm}\left(\xi_{\mp \varepsilon / 2, \varepsilon}^{ \pm}, \tau\right)\right)+O\left(\varepsilon^{2}\right)
\end{aligned}
$$

Then as $\widehat{f}^{ \pm}$is $C^{2}$, for $\varepsilon$ small enough, we obtain

$$
\begin{aligned}
\widehat{e}_{P}^{ \pm,(2)}(\mp \varepsilon / 2, \tau)= & \widehat{h}_{\varepsilon}^{\mp}(\tau) \exp \left(-2 \mathrm{i} \int_{-\varepsilon / 2}^{\varepsilon / 2} \lambda^{+}(y, \tau) d y\right) \\
& +\varepsilon^{2}\left(\partial_{x}^{2} \widehat{f}^{\mp}\left(\xi_{ \pm \varepsilon / 2, \varepsilon}^{\mp}, \tau\right) \mp \lambda^{+}(0, \tau) \partial_{x} \widehat{f}^{\mp}\left(\xi_{ \pm \varepsilon / 2, \varepsilon}^{\mp}, \tau\right)\right)+o\left(\varepsilon^{2}\right),
\end{aligned}
$$

so that

$$
\mathcal{F}_{t}\left(\mathcal{G}_{P}^{C} \circ \mathcal{G}_{P}^{C}\left\langle h_{\varepsilon}^{+}, h_{\varepsilon}^{-}\right\rangle\right)=\mathcal{F}_{t}\left(\mathcal{G}_{\Lambda}^{C} \circ \mathcal{G}_{\Lambda}^{C}\left\langle h_{\varepsilon}^{+}, h_{\varepsilon}^{-}\right\rangle\right)+\left\langle O\left(\varepsilon^{2}\right), O\left(\varepsilon^{2}\right)\right\rangle
$$


Let us recall that $C_{\Lambda, \varepsilon}^{C}=\sup _{\tau}\left|\exp \left(-2 \mathrm{i} \int_{-\varepsilon / 2}^{\varepsilon / 2} \lambda^{+}(y, \tau) d y\right)\right|$. Now using that $\partial_{x} \widehat{g}^{C, \pm}$ is bounded for $V$ and $V^{\prime}$ in $L^{\infty}\left(\Omega_{\varepsilon}^{ \pm}\right)$, there exists $c(\tau, \varepsilon, V)>0$ such that for $\varepsilon$ small enough

$$
\left.C_{P, \varepsilon}^{C} \leq \sup _{\tau} \mid \exp \left(-2 \mathbf{i} \int_{-\varepsilon / 2}^{\varepsilon / 2} \lambda^{+}(y, \tau) d y\right)+c(\tau, \varepsilon, V) \varepsilon^{2}\right) \mid
$$

Finally, there exists $C(\varepsilon, V)>0$ such that

$$
\sup _{\tau}\left\|\mathcal{F}_{t}\left(\mathcal{G}_{P}^{C} \circ \mathcal{G}_{P}^{C}\left\langle h_{\varepsilon}^{+}, h_{\varepsilon}^{-}\right\rangle\right)\right\|=C_{P, \varepsilon}^{C}\left\|\left\langle h_{\varepsilon}^{+}, h_{\varepsilon}^{-}\right\rangle\right\| \leq\left(C_{\Lambda, \varepsilon}^{C}+C(\varepsilon, V) \varepsilon^{2}\right)\left\|\left\langle h_{\varepsilon}^{+}, h_{\varepsilon}^{-}\right\rangle\right\|
$$

This concludes the proof.

\subsection{Asymptotic estimates of the contraction factor in the q-OSWR algorithm}

We consider now the quasi-Optimal Schwarz Waveform Relaxation (q-OSWR) method [23] and study its rates of convergence as a function of the order of the transmitting boundary conditions which are used. If we assume that $\phi^{ \pm,(0)}(\mp \varepsilon / 2, \cdot)$ and $\phi_{0}^{ \pm}$are some given functions, the q-OSWR algorithm reads as follows for an iteration $k \geq 1$ :

$$
\left\{\begin{array}{l}
P \phi^{ \pm,(k)}=0, \text { in } \Omega_{\varepsilon}^{ \pm} \times \mathbb{R}_{+}^{*}, \\
\phi^{ \pm,(k)}(\cdot, 0)=\phi_{0}^{ \pm}, \text {in } \Omega_{\varepsilon}^{ \pm}, \\
\left(\partial_{x}+i \Lambda^{ \pm, p}\right) \phi^{ \pm,(k)}( \pm \varepsilon / 2, \cdot)=\left(\partial_{x}+i \Lambda^{ \pm, p}\right) \phi^{\mp,(k-1)}( \pm \varepsilon / 2, \cdot) \text { in } \mathbb{R}_{+}^{*},
\end{array}\right.
$$

where $\Lambda^{ \pm, p}=\mathrm{Op}\left(\lambda^{ \pm, p}\right)$, for $p=1 / 2,0,-1 / 2 \ldots$ as similarly done in (26). Following [17], we set in $\Omega_{\varepsilon}^{ \pm}$

$$
P e_{P}^{O, \pm, p}=0 \text { on } \Omega_{\varepsilon}^{ \pm, p} \times \mathbb{R}_{+}^{*}, \quad\left(\partial_{x}+\text { i } \Lambda^{ \pm, p}\right) e_{P}^{O, \pm, p}( \pm \varepsilon / 2, t)=h_{\varepsilon}^{ \pm}(t) \text { on }\{ \pm \varepsilon / 2\} \times \mathbb{R}_{+}^{*},
$$

and we introduce the mapping

$$
\mathcal{G}_{P}^{O, p}:\left\langle h_{\varepsilon}^{+}, h_{\varepsilon}^{-}\right\rangle \mapsto\left\langle\left(\partial_{x}+\mathrm{i} \Lambda^{+, p}\right) e_{P}^{O,-, p}(\varepsilon / 2, \cdot),\left(\partial_{x}+i \Lambda^{-, p}\right) e_{P}^{O,+, p}(-\varepsilon / 2, \cdot)\right\rangle .
$$

We again want to estimate the asymptotic convergence rate of the q-OSWR domain decomposition method, when considering the system (41) for a transmission operator $\partial_{x}+i \Lambda^{ \pm, p}$. The q-OSWR method is now associated to the following approximate boundary-value problem on the error function $e_{\Lambda}^{O, \pm, p}$ :

$$
\left\{\begin{array}{l}
\left(\partial_{x}+i \Lambda^{\mp}\right) e_{\Lambda}^{O, \pm, p}=0, \text { in } \Omega_{\varepsilon}^{ \pm} \times \mathbb{R}_{+}^{*}, \\
\left(\partial_{x}+i \Lambda^{ \pm, p}\right) e_{\Lambda}^{O, \pm, p}( \pm \varepsilon / 2, t)=h_{\varepsilon}^{ \pm}(t), \text { on }\{ \pm \varepsilon / 2\} \times \mathbb{R}_{+}^{*} .
\end{array}\right.
$$

Then similarly to [10], we have

Theorem 2.2. Let us assume that $V$ is a smooth one-dimensional space-dependent potential and that $\lambda^{ \pm}$is approximated by

$$
\lambda^{ \pm} \approx \lambda^{ \pm, p}:=\sum_{j=0}^{p} \lambda_{1 / 2-j / 2}^{ \pm}
$$


with $p \in \mathbb{N}$. An asymptotic estimate in elliptic region of the contraction factor $C_{P, \varepsilon}^{O}$ of the mapping $\mathcal{G}_{P}^{O, p 2}$ (with $\left.\mathcal{G}_{P}^{O, p 2}:=\mathcal{G}_{P}^{O, p} \circ \mathcal{G}_{P}^{O, p}\right)$ defined by (43), for the fixed-point $q$-OSWR algorithm (41), is given by

$$
C_{P, \varepsilon}^{O} \approx C_{\varepsilon}^{O, p}=\sup _{\tau \in \mathcal{E}_{\tau}} L_{\varepsilon}^{O, p}(\tau)
$$

where

$$
L_{\varepsilon}^{O, p}(\tau) \approx c_{\varepsilon}^{p} \frac{1}{\left|\lambda_{1 / 2}^{+}(\varepsilon / 2, \tau) \lambda_{1 / 2}^{+}(-\varepsilon / 2, \tau)\right|^{p+1}} L_{\varepsilon}^{C, p}(\tau)
$$

In the hyperbolic zone, the frequencies also contribute to the q-OSWR convergence thanks to a contraction factor given by $c_{\varepsilon}^{p} \sup _{\tau \in \mathcal{H}_{\tau}}\left|\lambda_{1 / 2}^{+}(\varepsilon / 2, \tau) \lambda_{1 / 2}^{+}(-\varepsilon / 2, \tau)\right|^{-(p+1)}$. In the previous expressions, $c_{\varepsilon}^{p}$ is an $(\varepsilon, p, V)$-dependent positive real-valued constant. The principal symbol is given by (9) and $L_{\varepsilon}^{C, p}(\tau)$ designates (25). For a constant potential $V$, the fixed-point q-OSWR algorithm (41) converges in two iterations for the one-dimensional potential-free case, even without overlap $(\varepsilon=0)$.

The proof is very similar to the proof of Theorem 2.2 in [10], where was analyzed the q-OSWR convergence rate for the Schrödinger equation in imaginary time. Except for the symbols, the overall strategy and computational details are identical in both theorems. We here only give the sketch of the proof and again refer to [10] for more details.

Sketch of Proof. We first introduce the approximate problem (44) which gives us the approximate representation $\widehat{e}_{\Lambda}^{O, \pm, p}$ of the error $\widehat{e}_{P}^{O, \pm, p}$. Similarly to the analysis of the CSWR method, the aim is to derive an approximate convergence rate for the q-OSWR method, by introducing an analogous problem to (44). This can be expressed at the symbol level, and we know from (44)

$$
\left\{\begin{array}{l}
\left(\partial_{x}+i \lambda^{\mp}\right) \widehat{e}_{\Lambda}^{O, \pm, p}=0, \text { in } \Omega_{\varepsilon}^{ \pm} \times \mathbb{R}_{+}^{*}, \\
\left(\partial_{x}+i \lambda^{ \pm, p}\right) \widehat{e}_{\Lambda}^{O, \pm, p}( \pm \varepsilon / 2, t)=\widehat{h}_{\varepsilon}^{ \pm}(t), \text { on }\{ \pm \varepsilon / 2\} \times \mathbb{R}_{+}^{*},
\end{array}\right.
$$

which leads to the following expression

$$
\widehat{e}_{\Lambda}^{O, \pm, p}(x, \tau)=\alpha_{\varepsilon}^{ \pm, p}(\tau) \exp \left(-\mathrm{i} \int_{ \pm \varepsilon / 2}^{x} \lambda^{\mp}(y, \tau) d y\right),
$$

for some functions $\alpha_{\varepsilon}^{ \pm, p}$. By implementing the transmitting boundary conditions (second equation of system (46)), we get

$$
\widehat{e}_{\Lambda}^{O, \pm, p}(x, \tau)=\frac{\widehat{h}_{\varepsilon}^{ \pm}(\tau)}{i\left(\lambda^{ \pm, p}( \pm \varepsilon / 2, \tau)-\lambda^{\mp}( \pm \varepsilon / 2, \tau)\right)} \exp \left(-i \int_{ \pm \varepsilon / 2}^{x} \lambda^{\mp}(y, \tau) d y\right) .
$$

Now, we consider the mapping

$$
\mathcal{G}_{\Lambda}^{O, p}:\left\langle h_{\varepsilon}^{+}, h_{\varepsilon}^{-}\right\rangle \mapsto\left\langle\left(\partial_{x}+\mathrm{i} \Lambda^{+, p}\right) e_{\Lambda}^{O,-, p}(\varepsilon / 2, \cdot),\left(\partial_{x}+\mathrm{i} \Lambda^{-, p}\right) e_{\Lambda}^{O,+, p}(-\varepsilon / 2, \cdot)\right\rangle .
$$


By using (47), we get

$$
\begin{aligned}
\widehat{e}_{\Lambda}^{O, \pm, p,(2)}(x, \tau)= & \frac{\lambda^{\mp}( \pm \varepsilon / 2, \tau)+\lambda^{ \pm, p}( \pm \varepsilon / 2, \tau)}{\lambda^{ \pm, p}( \pm \varepsilon / 2, \tau)-\lambda^{\mp}( \pm \varepsilon / 2, \tau)} \times \frac{\widehat{h}_{\varepsilon}^{\mp}(\tau)}{i\left(\lambda^{\mp}, p(\mp \varepsilon / 2, \tau)-\lambda^{ \pm}(\mp \varepsilon / 2, \tau)\right)} \\
& \times \exp \left(-\mathrm{i} \int_{\mp \varepsilon / 2}^{ \pm \varepsilon / 2} \lambda^{ \pm}(y, \tau) d y\right) \exp \left(-\mathrm{i} \int_{ \pm \varepsilon / 2}^{x} \lambda^{\mp}(y, \tau) d y\right) .
\end{aligned}
$$

We recall that $\lambda^{-}=-\lambda^{+}$and $\lambda^{-, p}=-\lambda^{+, p}$, one can show that

$$
\begin{aligned}
& \mathcal{F}_{t}\left(\mathcal{G}_{\Lambda}^{O, p} \circ \mathcal{G}_{\Lambda}^{O, p}\left\langle h_{\varepsilon}^{+}, h_{\varepsilon}^{-}\right\rangle\right)(\tau) \\
& =\frac{\left[\lambda^{+, p}(\varepsilon / 2, \tau)-\lambda^{+}(\varepsilon / 2, \tau)\right]\left[\lambda^{+}(-\varepsilon / 2, \tau)-\lambda^{+, p}(-\varepsilon / 2, \tau)\right]}{\left[\lambda^{+, p}(\varepsilon / 2, \tau)+\lambda^{+}(\varepsilon / 2, \tau)\right]\left[\lambda^{+}(-\varepsilon / 2, \tau)+\lambda^{+, p}(-\varepsilon / 2, \tau)\right]} \\
& \times \exp \left(-2 \text { i } \int_{-\varepsilon / 2}^{\varepsilon / 2} \lambda^{+}(y, \tau) d y\right)\left\langle\widehat{h}_{\varepsilon}^{+}, \widehat{h}_{\varepsilon}^{-}\right\rangle(\tau) .
\end{aligned}
$$

From Proposition 2.3, we know that we have the following asymptotic control (for large $|-\tau+V| \gg 1$ ) of the remaining term

$$
\begin{aligned}
& \lambda^{+}( \pm \varepsilon / 2, \tau)-\lambda^{+, p}( \pm \varepsilon / 2, \tau)=\sum_{j=p+1}^{+\infty} \lambda_{1 / 2-j / 2}^{+}( \pm \varepsilon / 2, \tau) \\
& =\frac{1}{\left(\lambda_{1 / 2}^{+}( \pm \varepsilon / 2, \tau)\right)^{p}} \sum_{j=p+1}^{+\infty} \sum_{\ell=0}^{L_{j+1}} \frac{\mathcal{F}_{\ell}^{V,-j / 2}}{\left(\lambda_{1 / 2}^{+}\right)^{\ell}}( \pm \varepsilon / 2, \tau)=\mathcal{O}\left(\frac{1}{\left(\lambda_{1 / 2}^{+}( \pm \varepsilon / 2, \tau)\right)^{p}}\right) .
\end{aligned}
$$

Furthermore, we have

$$
\lambda^{+}( \pm \varepsilon / 2, \tau)+\lambda^{+, p}( \pm \varepsilon / 2, \tau)=2 \lambda_{1 / 2}^{+}( \pm \varepsilon / 2, \tau)+\mathcal{O}(1) .
$$

Collecting the various estimates, we conclude that

$$
\begin{aligned}
& \mathcal{F}_{t}\left(\mathcal{G}_{\Lambda}^{O, p 2}\left\langle h_{\varepsilon}^{+}, h_{\varepsilon}^{-}\right\rangle\right)(\tau)= \\
& \mathcal{O}\left(\frac{1}{\left(\lambda_{1 / 2}^{+}(\varepsilon / 2, \tau) \lambda_{1 / 2}^{+}(-\varepsilon / 2, \tau)\right)^{p+1}}\right) \times \exp \left(-2 \mathrm{i} \int_{-\varepsilon / 2}^{\varepsilon / 2} \lambda^{+}(y, \tau) d y\right)\left\langle h_{\varepsilon}^{+}, h_{\varepsilon}^{-}\right\rangle(\tau) .
\end{aligned}
$$

After truncating the symbolic expansion within the exponential term, an approximation rate of convergence for the q-OSWR DDM in both the elliptic zone, is given by

$$
C_{\varepsilon}^{O, p}=\sup _{\tau \in \mathcal{E}_{\tau}} L_{\varepsilon}^{O, p}(\tau)
$$

where

$$
L_{\varepsilon}^{O, p}(\tau) \approx c_{\varepsilon}^{p} \frac{1}{\left|\lambda_{1 / 2}^{+}(\varepsilon / 2, \tau) \lambda_{1 / 2}^{+}(-\varepsilon / 2, \tau)\right|^{p+1}} L_{\varepsilon}^{C, p}(\tau)
$$

where $L_{\varepsilon}^{C, p}(\tau)$ is given by $(25), p \in \mathbb{N}^{*}$, and $c_{\varepsilon}^{p}$ is an $\varepsilon$-, $p$ - and $V$-dependent positive realvalued constant. Unlike the CSWR method, the frequencies from the hyperbolic zone also contributes to the convergence of the q-OSWR method, thanks to the coefficients $1 /|\tau|^{p+1}$. 


\subsection{Convergence of the CSWR and q-OSWR algorithms}

We recall here two convergence theorems for the CSWR (5) and q-OSWR (41) algorithms when $V$ is constant, and which were proven in [23]. Unlike, Theorem 1.1, their proof relies on the calculation of the contraction factor, as proposed above.

Theorem 2.3. Let the initial guess $\left(h^{-}, h^{+}\right)$be in $\left(H^{3 / 4}(0, T)\right)^{2}$ and such that $h^{-}(0)=$ $\phi_{0}(-\varepsilon / 2)$ and $h^{+}(0)=\phi_{0}(\varepsilon / 2)$. Let $V$ be a real constant and suppose that $\tau_{\max }>0$ is such that $\mathcal{F}\left(e^{-t} h^{ \pm}\right)$vanishes outside of $\left[-\tau_{\max },+\infty\right)$ with $g^{+}=h^{+}-\phi^{+}(-\varepsilon / 2, \cdot)$ and $g^{-}=$ $h^{-}-\phi^{-}(\varepsilon / 2, \cdot)$. Then the iterates $\left(\phi^{-,(k)}, \phi^{+,(k)}\right)$ of the algorithm (5) converge in $L^{2}\left(\Omega_{\varepsilon}^{+} \times\right.$ $(0, T)) \times L^{2}\left(\Omega_{\varepsilon}^{-} \times(0, T)\right)$ to the solution of $(1)$.

The above theorem describes the convergence of the overlapping Schwarz waveform relaxation algorithm, stating that the convergence is at least linear and that it depends on the size of the overlapping region. For the CSWR, if the potential $V$ is constant, the approximate and the exact contraction factors coincide, that is $C_{P, \varepsilon}^{C}=C_{\Lambda, \varepsilon}^{C}$. The q-OSWR convergence theorem reads as follows:

Theorem 2.4. Let $V$ be a real constant. The algorithm (41) converges to the solution $\phi$ of (1) in two iterations for any initial guess $\phi^{+,(0)}$ and $\phi^{-,(0)}$, independently of the size of the overlap $(-\varepsilon / 2, \varepsilon / 2)$ for $\varepsilon>0$ if and only if

$$
\lambda^{+}=-\sqrt{-\tau+V}, \lambda^{-}=\sqrt{-\tau+V}
$$

When $V$ is constant, the OSWR algorithm then converges in two iterations, which corresponds to a contraction factor equal to 0 . When $V$ is non-constant, we expect a similar result. Thanks to the presented analysis (at least at high frequencies), the contraction factor can be shown to be arbitrarily small in the elliptic zone. However, notice that a rigorous proof for $V$ non-constant is yet to be established (work in progress).

\section{Numerical validation}

\subsection{Numerical approximation of the interior scheme}

In the one-dimensional case, we consider two bounded subdomains $\Omega_{a, \varepsilon}^{+}=(-a, b+\varepsilon / 2)$, $\Omega_{a, \varepsilon}^{-}=(b-\varepsilon / 2, a), a \in \mathbb{R}_{+}^{*}$ and with $\varepsilon>0$ a (small) parameter characterizing the overlapping region $\Gamma_{a, \varepsilon}=\Omega_{a, \varepsilon}^{+} \cap \Omega_{a, \varepsilon}^{-}=(b-\varepsilon / 2, b+\varepsilon / 2)$, and $\Omega_{a}=\Omega_{\varepsilon}^{+} \cup \Omega_{\varepsilon}^{-}=(-a, a)$. Finally, $b \pm \varepsilon / 2$ denotes the position of the interfaces. Notice that in the above analysis, we have taken $b=0$. The numerical scheme that we use is a second-order unconditionally $\ell^{2}$-stable Crank-Nicolson $(\mathrm{CN})$ scheme $[5,3]$. We define by $\Delta x$ the space step, and by $\Delta t_{n}$ the time-step at iteration $n$. We denote by $\phi_{j}^{ \pm, n,(k)}$ the solution in $\Omega_{\varepsilon}^{ \pm}$of the scheme at Schwarz iteration $k$, at time iteration $n$ and at $x_{j}^{ \pm}$, where the nodes $\left\{x_{j}^{ \pm}\right\}_{1 \leq j \leq N^{ \pm}}$are defined by: $x_{j}^{+}=-a+(j-1) \Delta x$, 
$x_{N^{+}}^{+}=b+\varepsilon / 2$ and $x_{j}^{-}=b-\varepsilon / 2+(j-1) \Delta x, x_{N^{-}}^{-}=a$. The CN scheme is defined as follows: for interior points in $(-a, a)$ and for $1<j<N^{ \pm}$, and $k \geq 1$ in $\Omega_{\varepsilon}^{ \pm}$

$$
\begin{aligned}
\frac{\phi_{j}^{ \pm, n+1,(k)}-\phi_{j}^{ \pm, n,(k)}}{\Delta t_{n}}= & \frac{i}{2 \Delta x^{2}}\left(\phi_{j+1}^{ \pm, n+1,(k)}-2 \phi_{j}^{ \pm, n+1,(k)}+\phi_{j-1}^{ \pm, n+1,(k)}\right) \\
& +\frac{i}{2 \Delta x^{2}}\left(\phi_{j}^{ \pm, n+1,(k)}-2 \phi_{j}^{ \pm, n+1,(k)}+\phi_{j-1}^{ \pm, n+1,(k)}\right) \\
& +\frac{i}{2} V_{j}^{ \pm}\left(\phi_{j}^{ \pm, n+1,(k)}+\phi_{j}^{ \pm, n,(k)}\right),
\end{aligned}
$$

where $V_{j}^{ \pm}=V\left(x_{j}^{ \pm}\right)$. The convergence criterion for the Schwarz DDM is given by

$$
\|\| \phi_{\mid \Gamma_{\varepsilon}}^{+, n_{T},(k)}-\phi_{\mid \Gamma_{\varepsilon}}^{-, n_{T},(k)}\left\|_{\infty, \Gamma_{\varepsilon}}\right\|_{L^{2}(0, T)} \leq \delta^{\mathrm{Sc}},
$$

with $\delta^{\mathrm{Sc}}=10^{-14}$ ("Sc" for Schwarz) and where $n_{T}=T / \Delta t$. When the convergence of the full iterative algorithm is obtained at Schwarz iteration $k^{\mathrm{cvg}}$, one gets the converged global solution $\phi^{\mathrm{cvg}}:=\phi^{\left(k^{\mathrm{cvg}}\right)}$ in $\Omega_{a}$. Let us remark that (64) is simple to implement since it is a standard CN scheme in each subdomain with homogeneous Dirichlet boundary conditions at the endpoint.

\subsection{Numerical validation of the CSWR}

The CSWR method is based on Dirichlet transmission conditions. At $x_{N^{+}}^{+}=b+\varepsilon / 2$, we impose $\phi_{N^{+}}^{+, n+1,(k)}+\phi_{N^{+}}^{+, n,(k)}=\phi_{j_{0}}^{-, n+1,(k-1)}+\phi_{j_{0}}^{-, n,(k-1)}$, and $j_{0}$ denotes the number of overlapping nodes, i.e. $\varepsilon=\left(j_{0}-1\right) \Delta x$. At $x_{1}=b-\varepsilon / 2$, we fix $\phi_{1}^{-, n+1,(k)}+\phi_{1}^{-, n,(k)}=\phi_{N^{+}-j_{0}}^{+, n+1,(k-1)}+\phi_{N^{+}-j_{0}}^{+, n,(k-1)}$. Finally at $x_{1}^{+}=-a$ and $x_{N^{-}}^{-}=a$, we set null Dirichlet boundary conditions. The $\ell^{2}$-stability and the second-order accuracy in space and time is straightforwards, as Dirichlet boundary conditions are used.

Test 1: potential $V=0$. The first test is devoted to two waves propagating in opposite directions from initial time $t=0$ to final time $T=1$. We follow the experiment given in [12]. The parameters for this first test are the following: $a=10, b=5 / 2$, with $N^{+}=312$ and $N^{-}=193$. The size of the overlapping zone is $\varepsilon=\Delta x$, corresponding to $j_{0}=2$. The time step is chosen equal to $\Delta t=0.2$. The initial data is given by

$$
\phi_{0}(x)=\exp \left(-\frac{1}{2}\left(\frac{b-a}{2}-x\right)^{2}\right) \exp \left(\mathrm{i} k_{0} x\right)+\exp \left(-\left(\frac{3 a}{8}+x\right)^{2}\right) \exp \left(-\mathrm{i} k_{0} x\right),
$$

with $k_{0}=4$, see Figure (1) (Left). In Figure (1) (Middle), we observe a good agreement between the reconstructed solution using the CSWR algorithm with the reference solution, obtained from a CN scheme on the global domain. From the above analysis (19), we get

$$
C_{P, \varepsilon}^{C}=C_{\Lambda, \varepsilon}^{C}=\sup L_{\Lambda, \varepsilon}^{C}\left(\tau_{\text {num }}\right),
$$

where $L_{\Lambda, \varepsilon}^{C}\left(\tau_{\text {num }}\right)=\left|\exp \left(-2 \mathbf{i} \varepsilon \lambda^{+}\left(\tau_{\text {num }}\right)\right)\right|$ and $\left|\tau_{\text {num }}\right| \in[1 / T, 1 / \Delta t]$. The supremum restricted to the elliptic zone, where $\tau_{\text {num }} \in[1 / T, 1 / \Delta t]$, exhibits a contraction factor $\exp (-\Delta x / \sqrt{T})<1$, as the supremum in this zone is actually reached at final time $T$. 

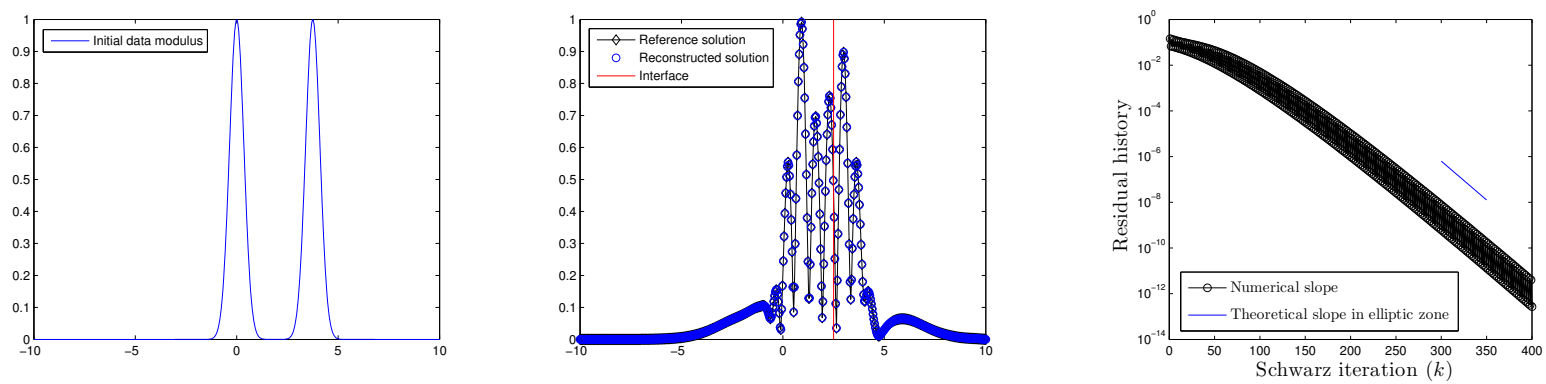

Figure 1: (Left) Initial data amplitude. (Middle) CSWR: reconstructed converged solution after iteration $k$ until convergence at final time $T$. (Right) Theoretical and numerical convergence rate comparison.

In Figure (1) (Right), we report the numerical convergence rate obtained by the CN scheme, and the theoretical convergence rates (33) in the elliptic zone, and we observe a relatively good agreement. Let us remark that the convergence rate is independent of the time-step $\Delta t$, but depends on the final time $T$ (as the supremum is reached at $1 / T$ ), as reported in Figure (2), where we observe that the smaller the final time $T$, the larger the convergence slope.

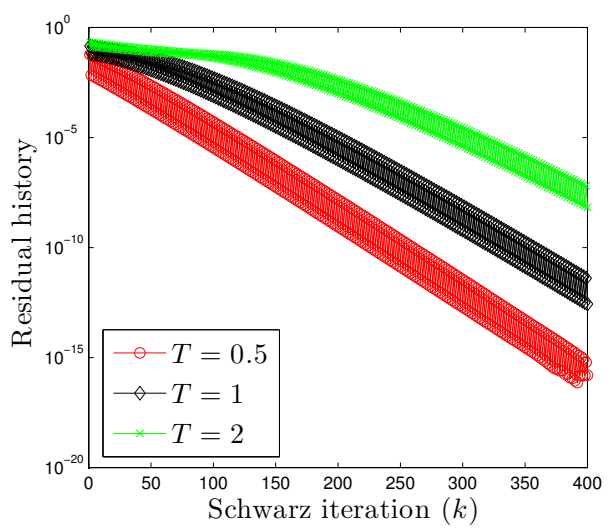

Figure 2: Convergence rates for different final times $T$.

Test 2: potential $V \neq 0$. The next test is also devoted to two waves propagating in opposite directions from time $t=0$ to $T=1$ with a positive space-dependent potential $V$. In this case, we expect the following estimate

$$
\begin{aligned}
& L_{\Delta x}^{C}\left(\tau_{\text {num }}\right) \approx \\
& \left|\left(\frac{\tau_{\text {num }}-V(-\Delta x / 2)}{\tau_{\text {num }}-V(+\Delta x / 2)}\right)^{1 / 2}\right|\left|\exp \left(-2 i \Delta x \int_{-\Delta x / 2}^{\Delta x / 2} \sqrt{-\tau_{\text {num }}+V(y)} d y\right)\right| .
\end{aligned}
$$

The parameters for the second experiment are as follows: $a=10, b=5 / 2$, with $N^{+}=312$, 
$N^{-}=193$ and the time step is $\Delta t=0.2$. The overlapping region is reduced to two nodes, $j_{0}=2$, such that $\varepsilon=\Delta x$. The initial data is given by

$$
u_{0}(x)=\exp \left(-4\left(\frac{b-a}{2}-x\right)^{2}\right) \exp \left(i k_{0} x\right)+\exp \left(-4\left(\frac{3 a}{8}+x\right)^{2}\right) \exp \left(-\mathrm{i} k_{0} x\right)
$$

with $k_{0}=4$. The space dependent potential given by $V(x)=-20 \exp \left(-5(x-b)^{2}\right)$. At the interface, we have $V(b) \approx-20$.
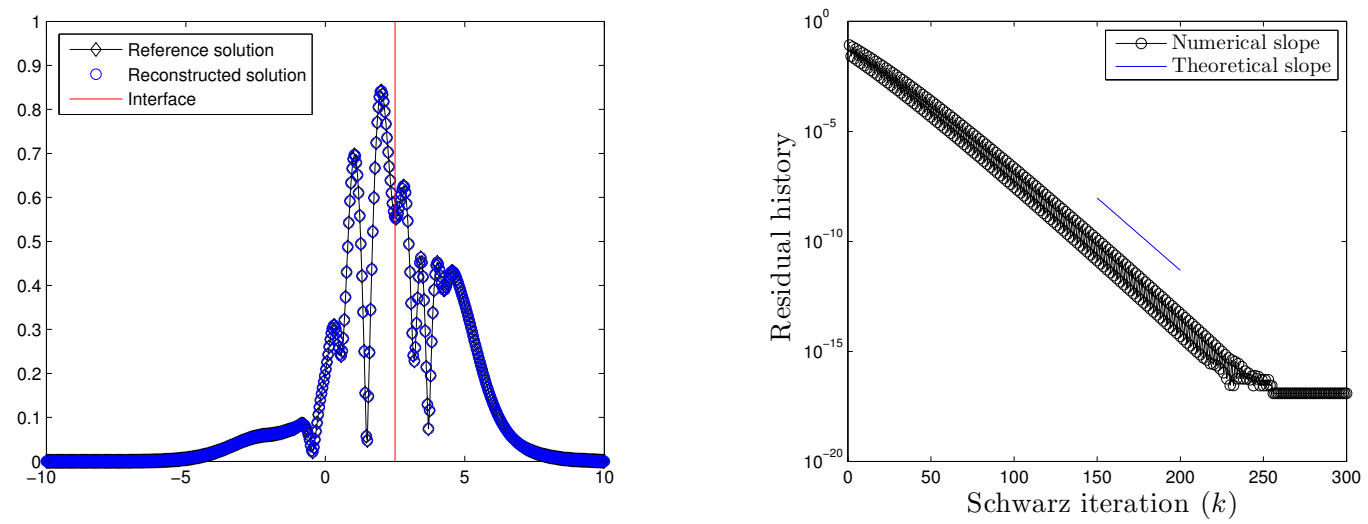

Figure 3: (Left): Reconstructed solution, and reference solution with space dependent potential $V$. (Right) Theoretical and numerical convergence rate comparison.

Since $V$ is negative, it is easy to see that the supremum of (57) is reached at $\tau_{\text {num }}=1 / \Delta t$, i.e.

$$
\begin{aligned}
& C_{P, \varepsilon}^{C} \approx \\
& \left|\left(\frac{1 / \Delta t-V(-\Delta x / 2)}{1 / \Delta t-V(+\Delta x / 2)}\right)^{1 / 2}\right|\left|\exp \left(-2 \mathrm{i} \Delta x \int_{-\Delta x / 2}^{\Delta x / 2} \sqrt{1 / \Delta t+V(y)} d y\right)\right|
\end{aligned}
$$

In Figure (3), we again observe a good agreement between the numerical and theoretical convergence rates. In Figure (4) (Middle) (resp. (Right)), the comparisons of the convergence rates are made between various time steps $\Delta t$ (resp. final time $T$ ), showing that the convergence rate, as expected is moderately dependent (resp. independent) of $\Delta t$ (resp. $T$ ), as $1 / \Delta t$ is relatively small compared to $V(b)=-20$.

In Figure (4), we observe that, after one Schwarz iteration with space dependent potential $V$, the solution is close to convergence unlike the case $V=0$.

\subsection{Numerical validation for $q-O S W R$}

The q-OSWR method involves DtN operators at the subdomain interfaces:

$$
\partial_{x} \phi^{ \pm,(k)}+\mathrm{i} \widetilde{\Lambda}^{ \pm, p}\left(x, t, \partial_{x}, \partial_{t}\right) \phi^{ \pm,(k)}=\partial_{x} \phi^{\mp,(k-1)}+\mathrm{i} \widetilde{\Lambda}^{ \pm, p}\left(x, t, \partial_{x}, \partial_{t}\right) \phi^{\mp,(k-1)},
$$



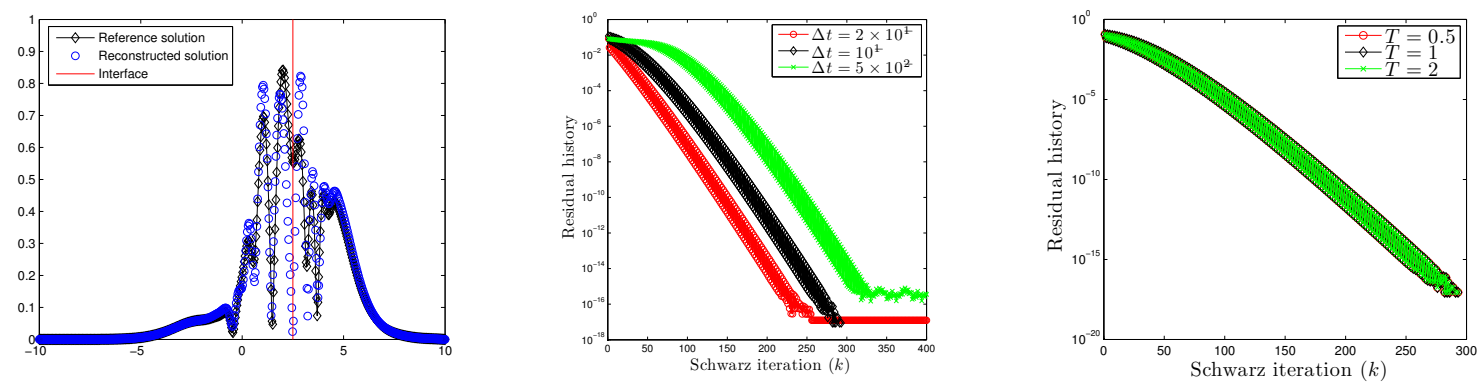

Figure 4: (Left) Reconstructed solution after 1 Schwarz iteration, and reference solution at final time $T=1$. (Middle) Convergence rates with different time-steps $\Delta t$ and space-dependent potential $V$ for CSWR. (Right) Convergence rates with different final times $T$, and space-dependent potential $V$ for CSWR.

with $\widetilde{\Lambda}^{ \pm, p}=\operatorname{Op}\left(\widetilde{\lambda}^{ \pm, p}\right)$ given by

$$
\left\{\begin{aligned}
\widetilde{\Lambda}^{+, 0}\left(x, t, \partial_{x}, \partial_{t}\right) \phi & =-e^{\mathrm{i} \pi / 4} \partial_{t}^{1 / 2} \phi \\
\widetilde{\Lambda}^{+, 1}\left(x, t, \partial_{x}, \partial_{t}\right) \phi & =-e^{\mathrm{i} \pi / 4} e^{\mathrm{i} \Phi} \partial_{t}^{1 / 2}\left(e^{-\mathrm{i} \Phi} \phi\right) \\
\widetilde{\Lambda}^{+, 4}\left(x, t, \partial_{x}, \partial_{t}\right) \phi & =\widetilde{\Lambda}^{+, 1} \phi+\frac{1}{4} d_{\mathbf{n}} V(x) e^{\mathrm{i} \Phi} I_{t}\left(e^{-\mathrm{i} \Phi} \phi\right)
\end{aligned}\right.
$$

and where the function $\Phi$ is defined in the linear case by $\Phi(x, t)=t V(x)$. The discretization of the nonlocal time operators are chosen as follows.

$$
\begin{aligned}
\partial_{t}^{1 / 2} f\left(t_{n}\right) & \approx \sqrt{\frac{2}{\Delta t}} \sum_{k=0}^{n} \beta_{n-k} f^{k}, \\
I_{t}^{1 / 2} f\left(t_{n}\right) & \approx \sqrt{\frac{\Delta t}{2}} \sum_{k=0}^{n} \alpha_{n-k} f^{k}, \\
I_{t} f\left(t_{n}\right) & \approx \frac{\Delta t}{2} \sum_{k=0}^{n} f^{k},
\end{aligned}
$$

where the sequence $\left(\beta_{n}\right)_{n \in \mathbb{N}}$ is such that $\beta_{0}=1$ and, for $n \geq 0$, and $\left(\gamma_{n}\right)_{n \in \mathbb{N}}$ are such that

$$
\left\{\begin{array}{l}
\left(\alpha_{0}, \alpha_{1}, \alpha_{2}, \alpha_{3}, \alpha_{4}, \alpha_{5}, \ldots\right)=\left(1,1, \frac{1}{2}, \frac{1}{2}, \frac{3}{8}, \frac{3}{8}, \ldots\right) \\
\beta_{k}=(-1)^{k} \alpha_{k}, \quad \forall k \geq 0 \\
\left(\gamma_{0}, \gamma_{1}, \gamma_{2}, \gamma_{3}, \ldots\right)=(1,2,2, \ldots)
\end{array}\right.
$$

The discretization (60)-(62) is designed to be consistent with the Crank-Nicolson scheme by using the associated generating function [5]. The semi-discrete q-OSWR-CN scheme for a 
two-domains decomposition method with a first-order ABC reads as follows

$$
\left\{\begin{array}{l}
\mathrm{i} \frac{\phi^{+, n+1,(k)}}{\Delta t}+\frac{1}{2}\left(\partial_{x}^{2} \phi^{+, n+1,(k)}+\partial_{x}^{2} \phi^{+, n,(k)}\right)+\frac{V(x)}{2}\left(\phi^{+, n+1,(k)}+\phi^{+, n,(k)}\right)=\frac{\phi^{+, n,(k)}}{\Delta t}, \text { in } \Omega_{a, \varepsilon}^{+}, \\
\left(\partial_{\mathbf{n}^{ \pm}}-e^{\mathrm{i} \pi / 4} \sqrt{\frac{2}{\Delta t}}\right) \phi_{ \pm \varepsilon / 2}^{ \pm, n+1,(k)}=g_{ \pm \varepsilon / 2}^{\mp, n+1,(k-1)}+\alpha_{ \pm \varepsilon / 2}^{ \pm, n,(k)}-\alpha_{ \pm \varepsilon / 2}^{\mp, n,(k-1)} \\
\phi^{ \pm, n+1,(k)}=0, \text { at } x=\mp a .
\end{array}\right.
$$

The outwardly directed unit normal vector to $\Omega_{a, \varepsilon}^{ \pm}$is denoted by $\mathbf{n}^{ \pm}= \pm 1$. We also set

$$
\begin{aligned}
& g_{ \pm \varepsilon / 2}^{\mp, n+1,(k-1)}=\partial_{\mathbf{n}^{ \pm}} \phi_{ \pm \varepsilon / 2}^{\mp, n+1,(k-1)}-e^{\mathrm{i} \pi / 4} \sqrt{\frac{2}{\Delta t}} \phi_{ \pm \varepsilon / 2}^{\mp, n+1,(k-1)}, \\
& \alpha_{ \pm \varepsilon / 2}^{\mp, n,(k)}=e^{\mathrm{i} \pi / 4} \sqrt{\frac{2}{\Delta t}} E_{ \pm \varepsilon / 2}^{n} \sum_{\ell=0}^{n} \beta_{n+1-\ell} \bar{E}_{ \pm \varepsilon / 2}^{\ell} \phi_{ \pm \varepsilon / 2}^{\mp, \ell,(k)}, \\
& E_{ \pm \varepsilon / 2}^{n}=\exp \left(-\mathrm{i} n \Delta t V_{ \pm \varepsilon / 2}\right) .
\end{aligned}
$$

Alternatively, a Neumann-to-Dirichlet approximation is possible. For the sake of simplicity, we assume that $V=0$, and the scheme reads as follows.

$$
\left\{\begin{aligned}
i \frac{\phi^{ \pm, n+1,(k)}}{\Delta t}+\frac{1}{2}\left(\partial_{x}^{2} \phi^{ \pm, n+1,(k)}+\partial_{x}^{2} \phi^{ \pm, n,(k)}\right)= & \frac{\phi^{ \pm, n,(k)}}{\Delta t}, \text { in } \Omega_{a, \varepsilon}^{+}, \\
= & -e^{i \pi / 4} \sqrt{\frac{\Delta t}{2}} \sum_{l=0}^{n+1} \alpha_{l} \partial_{\mathbf{n}^{+}} \phi_{ \pm \varepsilon / 2}^{ \pm, n+1-l,(k)}+\phi_{ \pm \varepsilon / 2}^{\mp, n+1,(k-1)} \\
\phi_{ \pm \varepsilon / 2}^{ \pm, n+1,(k)} & +e^{i \pi / 4} \sqrt{\frac{\Delta t}{2}} \sum_{l=0}^{n+1} \alpha_{l} \partial_{\mathbf{n}^{+}} \phi_{ \pm \varepsilon / 2}^{\mp, n+1-l,(k-1)} \\
= & 0, \text { at } x=\mp a .
\end{aligned}\right.
$$

We next show a partial stability result for (66).

Proposition 3.1. At Schwarz iteration $k=1$ and for $\varepsilon>0$, the semi-discrete scheme (66) is unconditionally $L^{2}$-stable. Moreover, for $\varepsilon=0$, the semi-scheme (66) is unconditionally $L^{2}$-stable at any Schwarz iteration.

Proof. We multiply i) $-\mathrm{i}\left(\bar{\phi}^{+, n+1,(k)}+\bar{\phi}^{+, n,(k)}\right) / 2\left(\operatorname{resp} . \quad-\mathrm{i}\left(\bar{\phi}^{-, n+1,(k)}+\bar{\phi}^{-, n,(k)}\right) / 2\right)$, ii) integrate on $\Omega_{\varepsilon}^{+}$(resp. on $\left.\Omega_{\varepsilon}^{-}\right)$and take the real part:

$$
\frac{1}{2 \Delta t}\left\|\phi^{+, n+1,(k)}\right\|_{L^{2}\left(\Omega_{\varepsilon}^{+}\right)}^{2}=\frac{1}{2 \Delta t}\left\|\phi^{+, n,(k)}\right\|_{L^{2}\left(\Omega_{\varepsilon}^{+}\right)}^{2}+\operatorname{Re}\left[i \frac{\bar{\phi}^{+, n+1,(k)}+\bar{\phi}^{+, n,(k)}}{2} \partial_{\mathbf{n}^{+}} \frac{\phi^{+, n+1,(k)}+\phi^{+, n,(k)}}{2}\right]_{+\varepsilon / 2}
$$

Set

$$
\phi_{+\varepsilon / 2}^{+, n+1 / 2,(k)}=\frac{\phi_{+\varepsilon / 2}^{+, n+1,(k)}+\phi_{+\varepsilon / 2}^{+, n,(k)}}{2}, \quad \phi_{-\varepsilon / 2}^{-, n+1 / 2,(k)}=\frac{\phi_{-\varepsilon / 2}^{-, n+1,(k)}+\phi_{-\varepsilon / 2}^{-, n,(k)}}{2}
$$


so that

$$
\frac{1}{2 \Delta t}\left\|\phi^{+, n+1,(k)}\right\|_{L^{2}\left(\Omega_{\varepsilon}^{+}\right)}^{2}=\frac{1}{2 \Delta t}\left\|\phi^{+, n,(k)}\right\|_{L^{2}\left(\Omega_{\varepsilon}^{+}\right)}^{2}+\operatorname{Re}\left[\mathbf{i} \bar{\phi}^{+, n+1 / 2,(k)} \partial_{\mathbf{n}^{+}} \phi^{+, n+1 / 2,(k)}\right]_{+\varepsilon / 2}
$$

and

$$
\frac{1}{2 \Delta t}\left\|\phi^{+, n+1,(k)}\right\|_{L^{2}\left(\Omega_{\varepsilon}^{+}\right)}^{2}=\frac{1}{2 \Delta t}\left\|\phi^{+, 0,(k)}\right\|_{L^{2}\left(\Omega_{\varepsilon}^{+}\right)}^{2}+\sum_{l=0}^{n} \operatorname{Re}\left[i \bar{\phi}^{l+1 / 2,(k)} \partial_{\mathbf{n}^{+}} \phi^{l+1 / 2,(k)}\right]_{+\varepsilon / 2}
$$

Similarly, we have

$$
\frac{1}{2 \Delta t}\left\|\phi^{-, n+1,(k)}\right\|_{L^{2}\left(\Omega_{\varepsilon}^{-}\right)}^{2}=\frac{1}{2 \Delta t}\left\|\phi^{-, 0,(k)}\right\|_{L^{2}\left(\Omega_{\varepsilon}^{+}\right)}^{2}+\sum_{l=0}^{n} \operatorname{Re}\left[\mathbf{i} \bar{\phi}^{-, l+1 / 2,(k)} \partial_{\mathbf{n}^{-}} \phi^{-, l+1 / 2,(k)}\right]_{-\varepsilon / 2}
$$

We set

$$
\mathbf{A}_{ \pm \varepsilon / 2}^{ \pm, n,(k)}=\sum_{l=0}^{n} A_{ \pm \varepsilon / 2}^{ \pm, l,(k)}=\sum_{l=0}^{n} \operatorname{Re}\left[\mathbf{i} \bar{\phi}^{ \pm, l+1 / 2,(k)} \partial_{\mathbf{n}^{ \pm}} \phi^{ \pm, l+1 / 2,(k)}\right]_{ \pm \varepsilon / 2}
$$

and

$$
\zeta=e^{i \pi / 4} \sqrt{\frac{\Delta t}{2}}
$$

The transmission conditions can now also read

$$
\phi_{ \pm \varepsilon / 2}^{ \pm, n+1,(k)}+\zeta \sum_{l=0}^{n+1} \alpha_{l} \partial_{\mathbf{n}^{ \pm}} \phi_{ \pm \varepsilon / 2}^{ \pm, n+1-l,(k)}=\phi_{ \pm \varepsilon / 2}^{\mp, n+1,(k-1)}+\zeta \sum_{l=0}^{n+1} \alpha_{l} \partial_{\mathbf{n}^{+}} \phi_{ \pm \varepsilon / 2}^{\mp, n+1-l,(k-1)}
$$

Now at $k=0$, we assumed that $\phi_{+\varepsilon / 2}^{+, n,(0)}=\phi_{-\varepsilon / 2}^{-, n,(0)}=0$, and then, for $k=1$

$$
\phi_{ \pm \varepsilon / 2}^{ \pm, n+1,(1)}+\zeta \sum_{l=0}^{n+1} \alpha_{l} \partial_{\mathbf{n}^{ \pm}} \phi_{ \pm \varepsilon / 2}^{ \pm, n+1-l,(1)}=0
$$

Finally, by using Theorem 4 from [5], we deduce

$$
\operatorname{Re}\left[i \bar{\phi}^{ \pm, l+1 / 2,(l)} \partial_{\mathbf{n}^{ \pm}} \phi^{ \pm, l+1 / 2,(1)}\right]_{ \pm \varepsilon / 2} \leq 0
$$

and the corresponding schemes in $\Omega_{\varepsilon}^{+}$and $\Omega_{\varepsilon}^{-}$, which are then purely non-reflecting, are then unconditionally $L^{2}$-stable if $k=1$.

Note that for $\varepsilon=0$, we directly get from the transmission conditions that

$$
\phi_{0}^{ \pm, n+1,(k)}+\zeta \sum_{l=0}^{n+1} \alpha_{l} \partial_{\mathbf{n}^{ \pm}} \phi_{0}^{ \pm, n+1-l,(k)}=\phi_{0}^{\mp, n+1,(k-1)}+\zeta \sum_{l=0}^{n+1} \alpha_{l} \partial_{\mathbf{n}^{ \pm}} \phi_{0}^{\mp, n+1-l,(k-1)} .
$$


In other words for all $k \geq 1$, we get

$$
\operatorname{Re}\left[\mathrm{i} \bar{\phi}^{ \pm, l+1 / 2,(k)} \partial_{\mathbf{n}^{ \pm}} \phi^{ \pm, l+1 / 2,(k)}\right]_{0} \leq 0 .
$$

This ensures the $L^{2}$-stability of the semi-discrete scheme.

The proof of stability at any Schwarz iteration when $\epsilon>0$ requires additional assumptions and is not presented in this paper.

Based on this discretization, we now validate the theoretical rate of convergence for the q-OSWR method. Let us define $T_{\ell}=T_{0} / 2^{\ell}$, with $\ell=-2,-1,0,1,2$. According to the above analysis, we have $C_{p, \ell}^{(\mathrm{OSWR})} \approx c_{\varepsilon}^{p} C_{\ell}^{(\mathrm{CSWR})} T_{\ell}^{(p+1) / 2}$, where $p \in \mathbb{N}$ designates the order of approximation of the "transparent" operator $\Lambda^{+}$and $c_{\varepsilon}^{p}$ is an unknown $(\varepsilon, p)$-dependent constant. In order to numerically validate this result, we first observe that

$$
\log C_{p, \ell}^{(\mathrm{OSWR})} \approx \log C_{\ell}^{(\mathrm{CSWR})}+\log \left(c_{\varepsilon}^{p} T_{0}^{(p+1) / 2}\right)-\ell(p+1) \log (2) / 2 .
$$

We next numerically evaluate

$$
\left|\log C_{p, \ell}^{(\mathrm{OSWR})}-\log C_{\ell}^{(\mathrm{CSWR})}\right|
$$

as a function of $\ell$. For $p=0$, the transparent transmission operator is approximated by

$\partial_{t}^{1 / 2}$ the expected slope is $-\log (2) / 2$, when plotting $\log C_{p, \ell}^{(\mathrm{OSWR})}$ as a function of $\ell$. The numerical data are as follows: $N^{+}=641, N^{-}=385, a=-20, \Delta t=0.1, T_{0}=1, \varepsilon=\Delta x$, $b=4$ and $k_{0}=4$, and

$$
\phi_{0}(x)=\exp \left(-4\left(\frac{b-a}{2}-x\right)^{2}\right) \exp \left(\mathrm{i} k_{0} x\right)+\exp \left(-4\left(\frac{3 a}{8}+x\right)^{2}\right) \exp \left(-\mathrm{i} k_{0} x\right)
$$

and $V(x)=5 \exp \left(-5(x-b)^{2}\right)$. In Figure 5, we compare the theoretical and numerical slopes for $p=0$. We observe that the numerical convergence slope is close to the theoretical one, i.e. $-\log (2) / 2$. To validate the theoretical slopes for larger values of $p$, it is necessary to develop a much heavier numerical machinery not presented in this paper.

\section{Conclusion}

The paper was devoted to the analysis of the rate of convergence of the Schwarz Waveform Relaxation domain decomposition methods applied to the linear Schrödinger equation with space dependent potential. Approximations of the theoretical convergence rate have been stated for both the CSWR and the q-OSWR algorithms by using pseudo-differential. In addition, they were also numerically validated. In a forthcoming paper, we will analyze the convergence rate of SWR in higher dimensions and for more subdomains.

\section{Acknowledgments.}

X. Antoine thanks the support of the french ANR grants "Bond" (ANR-13-BS01-000901) and ANR-12-MONU-0007-02 BECASIM (Modèles Numériques call). E. Lorin thanks NSERC for the financial support via the Discovery Grant program. 


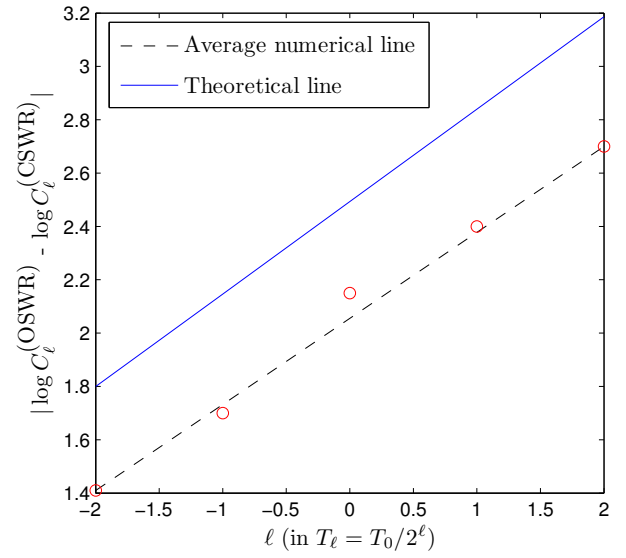

Figure 5: Theoretical and numerical convergence rate for q-OSWR with $p=0$. 


\section{References}

[1] M. Al-Khaleel, A.E. Ruehli, and M.J. Gander. Optimized waveform relaxation methods for longitudinal partitioning of transmission lines. IEEE Transactions on Circuits and Systems, 56:1732-1743, 2009.

[2] S. Alinhac and P. Gérard. Pseudo-differential operators and the Nash-Moser theorem, volume 82 of Graduate Studies in Mathematics. American Mathematical Society, Providence, RI, 2007. Translated from the 1991 French original by Stephen S. Wilson.

[3] X. Antoine, W. Bao, and C. Besse. Computational methods for the dynamics of the nonlinear Schrödinger/Gross-Pitaevskii equations. Computer Physics Communications, 184(12):2621-2633, 2013.

[4] X. Antoine and C. Besse. Construction, structure and asymptotic approximations of a microdifferential transparent boundary condition for the linear Schrödinger equation. Journal de Mathématiques Pures et Appliquées, 80(7):701-738, 2001.

[5] X. Antoine and C. Besse. Unconditionally stable discretization schemes of non-reflecting boundary conditions for the one-dimensional Schrödinger equation. J. Comput. Phys., 188(1):157-175, 2003.

[6] X. Antoine, C. Besse, and P. Klein. Absorbing boundary conditions for the onedimensional Schrödinger equation with an exterior repulsive potential. J. Comput. Phys., 228(2):312-335, 2009.

[7] X. Antoine, C. Besse, and P. Klein. Absorbing boundary conditions for the twodimensional Schrödinger equation with an exterior potential. Part I: construction and a priori estimates. Math. Models Methods Appl. Sci., 22(10):1250026, 38, 2012.

[8] X. Antoine, C. Besse, and P. Klein. Absorbing boundary conditions for the twodimensional Schrödinger equation with an exterior potential. Part II: discretization and numerical results. Numer. Math., 125(2):191-223, 2013.

[9] X. Antoine and E. Lorin. Lagrange - Schwarz waveform relaxation domain decomposition methods for linear and nonlinear quantum wave problems. Applied Math. Lett., 57, 2016.

[10] X. Antoine and E. Lorin. An analysis of Schwarz waveform relaxation domain decomposition methods for the imaginary-time linear Schrödinger and Gross-Pitaevskii equations. Numer. Math., 2017.

[11] X. Antoine and E. Lorin. Asymptotic estimates of the convergence of classical Schwarz waveform relaxation domain decomposition methods for two-dimensional stationary quantum waves. Revision. ESAIM: Numerical Analysis and Mathematical Modeling (M2AN), 2017. 
[12] X. Antoine, E. Lorin, and A. Bandrauk. Domain decomposition method and highorder absorbing boundary conditions for the numerical simulation of the time dependent Schrödinger equation with ionization and recombination by intense electric field. J. Sc. Comput., 64(3):620-646, 2015.

[13] X. Antoine, E. Lorin, and Q. Tang. A friendly review to absorbing boundary conditions and perfectly matched layers for classical and relativistic quantum wave equations. Molecular Physics, 115(15-16):1861-1879, 2017.

[14] C. Besse and F. Xing. Schwarz waveform relaxation method for one dimensional Schrödinger equation with general potential. Numer. Algo., pages 1-34, 2016.

[15] V. Dolean, P. Jolivet, and F. Nataf. An Introduction to Domain Decomposition Methods: Algorithms, Theory, and Parallel Implementation, volume 144. SIAM, 2015.

[16] B. Engquist and A. Majda. Absorbing boundary conditions for the numerical simulation of waves. Math. Comp., 31(139):629-651, 1977.

[17] M. Gander and L. Halpern. Optimized Schwarz waveform relaxation methods for advection reaction diffusion problems. SIAM J. on Numer. Anal., 45(2):666-697, 2007.

[18] M.J. Gander. Optimal Schwarz waveform relaxation methods for the one-dimensional wave equation. SIAM J. Numer. Anal., 41:1643-1681, 2003.

[19] M.J. Gander. Optimized Schwarz methods. SIAM J. Numer. Anal., 44:699-731, 2006.

[20] M.J. Gander, L. Halpern, and F. Nataf. Optimal convergence for overlapping and nonoverlapping Schwarz waveform relaxation. page 1999.

[21] M.J. Gander, F. Kwok, and B.C. Mandal. Dirichlet-neumann and neumann-neumann waveform relaxation for thewave equation. Lecture Notes in Computational Science and Engineering, 104:501-509, 2016.

[22] L. Halpern and J. Rauch. Error analysis for absorbing boundary conditions. Numer. Math., 51(4):459-467, 1987.

[23] L. Halpern and J. Szeftel. Optimized and quasi-optimal Schwarz waveform relaxation for the one-dimensional Schrödinger equation. Mathematical Models and Methods in Applied Sciences, 20(12):2167-2199, 2010.

[24] L. Hörmander. Linear Partial Differential Operators, volume 116. Springer, 2013.

[25] J-L. Lions and E. Magenes. Problèmes aux limites non homogènes et applications. vol. 1. 1968.

[26] E. Lorin. Schwarz waveform relaxation domain decomposition methodology for the N-body time-independent and time-dependent Schrödinger equation. Submitted, 2017. 
[27] E. Lorin, X. Yang, and X. Antoine. Frozen gaussian approximation based domain decomposition methods for the linear and nonlinear Schrödinger equation beyond the semi-classical regime. J. Comput. Phys., 315:221-237, 2016.

[28] B.C. Mandal. A time-dependent Dirichlet-Neumann method for the heat equation. Lecture Notes in Computational Science and Engineering, 98:467-475, 2014.

[29] L. Nirenberg. Lectures on Linear Partial Differential Equations. American Mathematical Society, Providence, R.I., 1973. 